\title{
Mathematical Nanotechnology: Quantum Field Intentionality
}

\author{
Francisco Bulnes \\ Research Department in Mathematics and Engineering, Technological Institute of High Studies of Chalco, \\ Federal Highway Mexico-Cuautla s/n Tlapala "La Candelaria" Chalco, Mexico City, Mexico \\ Email: francisco.bulnes@tesch.edu.mx
}

Received July 29, 2013; revised August 29, 2013; accepted September 15, 2013

Copyright (C) 2013 Francisco Bulnes. This is an open access article distributed under the Creative Commons Attribution License, which permits unrestricted use, distribution, and reproduction in any medium, provided the original work is properly cited.

\begin{abstract}
Considering the finite actions of a field on the matter and the space which used to infiltrate their quantum reality at level particle, methods are developed to serve to base the concept of "intentional action" of a field and their ordered and supported effects (synergy) that must be realized for the "organized transformation" of the space and matter. Using path integrals, these transformations are decoded and their quantum principles are shown.
\end{abstract}

Keywords: Intentional Action; Field Infiltration; Hyper-Reality; Synergic Action; Events Synchronization; Synergic Operators; Cyber-Quantum Algebra; Perception Integral Transforms; Feynman-Bulnes Integrals; Newman Dimension

\section{Introduction}

The nanotechnology will bring new paradigms of the scientific thought and will change our limited vision of the world since the real causes of any phenomenon initiate from an atomic scale, the fifth part of the one hundred millionth part of a meter $\left(\approx(1 / 5) 10^{-9}\right.$ meters) (and even subatomic if we want to understand the communication and transference of the states of energy between atoms). Only in these small dimensions, the real reality exists, since everything what our senses can receive there are ordered holograms that obey a cosmic perception that has been developed according to a field conscience that spills their creative intentions from a quantum zone.

We consider a set of particles in the space $\mathrm{E}$, under certain law of movement defined by their Lagrangian $L$, we have that the action defined by a field that acts with this movement law and that causes it, as is defined by the $\operatorname{map}[1]$ :

$$
\mathfrak{s}: \mathrm{TE} \rightarrow \mathbb{R},
$$

with rule of correspondence

$$
\mathfrak{I}(x(s))=\operatorname{Flux} L(x(s)) x(s),
$$

We can establish that the global action in a particle system with instantaneous action can be re-interpreted locally as a permanent action of the field considering the synergy of the instantaneous temporary actions under this permanent action of the field. This passes to the following principle:

Principle 1. The temporary or instantaneous action on a global scale can be measured like a local permanent action

The previous principle together with certain laws of synchronicity of events in the space time will shape one of the governing principles of the nanotechnology, why?

Because, at microscopic level, the permanence of a field is constant, in proportion to the interminable state and permanence of energy that exist in the atoms. As a result of this, a nano-technological process will be directed to the manipulation of the microstructures of the components of the matter using this principle of "intentional action". The time at quantum level is a time measured in ns (nano-seconds) which is compared to the time measured in the scales that measure the visible effects in any physical process; they turn out to be almost nonexistent or void. Nevertheless, this nullity characterizes the flatness of the space time to these quantum scales (the time stops existing like an entity not separated from the space), and is where the field finds permanence shaped by a quantum sea of light of bosons. Then any macroscopic temporary action is measured by an action that is born from a permanent field at local level, and everywhere, since it belongs to this quantum light sea. 
Let $G$ be the Lie group of actions defined by their automorphisms $\sigma \in G$, such that these actions from $G$, are defined in their algebra $\mathrm{g}$, through the diffeomorphism $\exp (t X), \forall X \in \mathbb{g}$ [2]. If we consider the algebra $\mathfrak{q}_{e} \subset \mathfrak{g}$, that is to say, find their local application in their algebra $\mathfrak{a}$, then we can access to the quantum zone of the nullity where the temporal effects are annulled. Then in $\mathfrak{g}_{e}, \partial^{\mu} X_{\mu}(x)=0, \forall x \in \mathbb{M} \quad$ [3].

Then supposing that the field $X$ can control under finite actions like the described for $\mathfrak{I}$, and under the established principle, we can execute an action on a microstructure always and when the sum of the actions of all the particles is major than their algebraic sum (to give an order to only one particle so that the others continue it).

How to obtain this combined effect of all the particles that move and that is wanted to realize a coordinated action (of tidy effect) and simultaneously (synchronicity), with the only effect?

Inside the universe of minimal trajectories that satisfies the variation functional [1], we can choose a $\gamma_{t} \in \Omega(\Gamma)$, such that

$$
\operatorname{Exe} \mu_{t}=\int_{\gamma_{t}}\left(\int_{p_{1}}^{p_{2}} L(x(s)) \mathrm{d}(x(s))\right) \mu_{\gamma_{t}},
$$

which is not arbitrary, since we can define any action on $\gamma_{t}$, like

$$
\widetilde{J}_{\gamma_{t}}=\int_{p_{1}}^{p_{2}} L(x(s), \dot{x}(s), s) \mathrm{d} s,
$$

that is to say, there is an intention defined by the field action that infiltrates into the whole space of the particles influencing or "infecting" the temporary or instantaneous actions making that the particles arrange themselves all and with added actions not in the algebraic sense, but in the holistic sense. This action is the "conscience" that has the field to exercise their action in "intelligent" form that is to say, in organized form through their path integrals like the described by the classical Feynman integrals. Then extending the above mentioned integral to the whole space $\Omega(\Gamma)$, we have the synergic principle of the total field $X$,

$$
\mathfrak{I}_{\text {TOTAL }} \geq \sum_{j} \int_{\gamma_{t}} \mathfrak{I}_{j}(x(s)) \mathrm{d}(x(s)),
$$

the length and breadth of $\mathbb{E}$.

The order conscience is described by the operator of execution of a finite action of a field $X$, on a target (region of space that must be infiltrated by the action of the field which is that for which we realize our re-walked $\Omega(\Gamma)$ ).

Likewise if we choose a geodesic $\gamma_{t}$, of the field $X$, on which an action will be applied $\mathfrak{I}$, measurable in the Lagrange ambience, we have that their execution is as given in (3). The action must be realized in form supported along the object to which is required apply the transformation due to $X$.

How to measure this transference of conscience of transformation due to the field $X$, on an object defined by a portion of the space $\Omega(\Gamma)$ ? Which is the limit of this supported action or transference of conscience so that it feeds the effect in the portion of the space $\Omega(\Gamma)$, and the temporary or instantaneous actions for every particle $x^{i}$, are founded on only one global synergic action on $\Omega$ ?

We measure this transference of conscience (or intention) of $X$, on a particle $x(s)$, through the value of the integral of the spilled intelligence (path integral) given as [4]:

$$
\begin{aligned}
& \left\langle\tau_{\alpha} X(x(s)), x_{\gamma}\right\rangle \\
& =\int_{\Omega(\Gamma)} \mathfrak{I}\left(x_{\delta} \circ x_{\sigma} \circ x_{\delta}^{-1}\right)\left(\phi_{\sigma}\left(x_{\eta}\right) \phi_{\sigma}\left(x_{\gamma}\right) \phi_{\sigma}^{-1}\left(x_{\eta}\right)\right) \mu_{\sigma},
\end{aligned}
$$

We left at level conjecture and based on our researches of nanotechnology and advanced quantum mechanics that a sensor for the quantum sensitization of any particle that receives an instruction given by a field $X$, must satisfy the inequality of Hilbert type [5], for this transference of conscience is defined in (6) on the region $\Omega(\Gamma)$, to know [4]:

$$
\begin{aligned}
& \left\langle\tau_{\alpha} X(x(s)), t_{\gamma}\right\rangle \\
& \leq\left\|\log \phi_{\sigma}\left(x_{\eta}\right)\right\|^{a}\left\|\log \mathfrak{I}\left(x_{\sigma}\right)\right\|^{b}, \text { con } a=b=2
\end{aligned}
$$

The conscience transference demands a synchronization of events that obeys to an effect of simultaneity and coordination of temporary actions on a set of particles that must behave under the same intensity that could be programmed through "revisited" path integrals [6], producing a joint effect called synergy [7].

In the above mentioned process, the time and the space are interchangeable in the quantum zone as we can observe it in the integrals (3), and where a particle will be and when it will be there, are aspects that go together. Therefore not only the energy must be quantized but also the magnitude "distance", whose discrete length quantity must perform a low order to $12^{-13} \mathrm{~cm}$ (spin relation between two connected virtual particles [8]). The energy is to form a particle for an observer who lies in a space time $M r$.

The above mentioned particle will disappear to the view of the same observer if this one lives in one of the coordinated axes of the space $M$, if the observer is not in any of these axes. On the other hand, for an observer in the region $\Omega(\Gamma)$, this one will see the particles as trajectories that fill the space as instantons with time [9]. Out of these ambiences the alone observer perceives a cloud of energy that is permanent.

Any transformation that is wanted to realize a space, 
takes as constant the same energy that comes from the permanent field of the matter and which is determined by the quantum field of the constituents particles $x(s)$, of the space and matter. If we want to define a conscience in the above mentioned field, that is to say, an action that involves an intention is necessary to establish this intention inside the argument of the action.

Likewise, if $x(s) \in \Omega$, and $\mathfrak{I}(x(s))$, is their action due to a field of particles $X$, and is a spilled intention defined by (3) showing the length and breadth of the space $M$, such that to satisfy (5), for all the possible trajectories that fill $\Omega$, we have

$$
\mathfrak{I}_{\text {TOTAL }} \geq \int_{E^{-}}^{E^{+}}\left\{\sum_{j} \int_{\gamma_{t}} \mathfrak{I}_{j}(x(s)) \mathrm{d}(x(s))\right\} \mathrm{d} \mu,
$$

where the total action is an intentional action (for all the infinity of paths $\gamma_{t}$, that define to $\Gamma$ )

$$
\begin{aligned}
\mathfrak{I}_{\text {TOTAL }} & =\int_{\Gamma}\left\{\mathfrak{I}\left[\int_{\Omega(\Gamma)} O_{c}(x(t)) \mathrm{d}(x(s))\right] \mu_{t}\right\} \\
& =\left(E^{+}-E^{-}\right) \int_{-\infty}^{+\infty}\left\{\int_{\Omega(\Gamma)} \mathfrak{I}(x(s)) d x(s)\right\} \mathrm{d} t,
\end{aligned}
$$

where the energy factor $E^{+}-E^{-}$, represents the energy needed by the always present force to realize the action and $O_{c}$, is the conscience operator who defines the value or record of the field $X$, (direction), on every particle of the space $\Omega(\Gamma)$, which along their set of trajectories $\Gamma$, realizes the action of permanent field $\mathfrak{J}_{O_{c}}$, which is

$$
\mathfrak{I}_{O_{c}}(x(s))=\int_{x(\mathrm{M})} O_{c}(x(s)) \mathrm{d} x(s),
$$

where the operator $\mathfrak{I}_{O_{c}}$, invests an energy quasi infinite, encapsulated in a microscopic region of the space (quantum space $\mathcal{M}$ ), and with applications and influence in an unlimited space of the sub-particles (boson space). Likewise a photon of certain class $x(s)$, will be generated by the quantum field (if it manages to change its field spin) and will be moved for the intention on a trajectory $\Gamma$, by the path integral

$$
\mathfrak{I}\left(\mathfrak{I}_{O_{c}}(x(s))\right)=\int_{\Gamma}\left[\int_{X(C)} O_{c}(x(s)) \mathrm{d}(x(s)) \mu_{\Gamma}\right],
$$

Interesting applications of the formula (9) to nanosciences will happen at the end of the present chapter. Also it will be demonstrated that (9) is a quantum integral transform of bundles or distortions of energy in the space-time if it involves a special kernel. The bundle stops existing if certain intention is applied (path integral transform). The operator $O_{c}$, involves a connection of the tangent bundle of the space of trajectories $\Omega(\Gamma)$.
The integral (11) will determine on certain hypotheses the interdependence between the material, quantum and virtual realities in $\mathrm{M}$, (see formula (44)).

Then under this perspective we can define the quantum zone of the space-time as susceptible to the application of intentions which establish statistical weights in the elected trajectories of $\Omega(\Gamma)$ to do it. These statistical weights are born of considering the existence of diffeomorphisms between elements of the topological group $G$, that is subjacent in the space $M$ (structure of the operators of the actions of the field $X$, in the space $\mathbb{M}$ ) that wants to transform and corresponding algebra $\mathfrak{g}$ (Lie algebra) of this group that records the operators of the actions of the field locally (space time of every particle).

Definition 1.1. [12]. The space-time in the quantum zone $\mathcal{M}$, is the spatial region of the flux of permanent field (region of application of the field) where are the diffeomorphic correspondences which are subjacent to the relations between the macroscopic effects, and the quantum phenomena of $\mathcal{M}$. The diffeomorphisms come given for a uni-parametric group $\left\{\mathrm{e}^{-\mathrm{i} \alpha_{t}(X)}\right\}$, of the quantum field of the space $\mathcal{M},[10,11]$ (generator of quantum isometries) which defines the Lagrangian special $O_{c}$, of a conscience operator defined for an integral of action [12], as given by (10).

The macroscopic effects, that is to say, the effects generated in the space $M$, come from causes that take place from the set of automorphisms of the group $G$ (topological group) in which the cause - effect laws are established between elements of $G$, evaluated in every particle.

Considering a particle system $p_{1}, p_{2}, \cdots$, in a space time $M \cong \mathbb{R}^{4}$. Let $x(t) \in \Omega(\Gamma) \subset \mathbb{R}^{3} \times I_{t}$, being a trajectory which predetermines a position $x \in \mathbb{R}^{3}$, for all time $t \in I_{t}$. A field $X$ that infiltrates their action to the whole space of points predetermined by all the trajectories $x_{1}(t), x_{2}(t), x_{3}(t), \cdots \in \Omega(\Gamma)$, is the field that pre-determines the points $\phi_{i}\left(x_{i}(t)\right)$, which are fields whose determination is given by the action of the field $X$, and evaluated in the position of every particle. Every point has a defined force by the action $\mathfrak{I}$, of $X$, along the geodesic $\gamma_{t}$, and determined direction by their tangent bundle given for $T \mathcal{K}^{1}(\Omega(\Gamma)), \forall \Phi \in \mathcal{X}^{1}(\Omega(\Gamma))$, that is to say, the field provides direction to every point $\phi_{i}$, because their tangent bundle has a spinor bundle $\mathrm{S}$ [13], where the field $X$ comes given

$$
X=\left.\sum_{i} \phi^{i} \frac{\partial}{\partial \phi^{i}}\right|_{\left(x^{i}, \phi^{i}\right)}, \quad \forall \phi_{1}, \phi_{2}, \phi_{3}, \cdots \in \mathcal{X}^{1} \text {, on every }
$$

particle $p_{i}=x_{i}(t),(i=1,2, \cdots)$. Then to direct an intention we have the map or connection:

$$
\nabla^{\mathfrak{J}}: T \Omega(\Gamma) \rightarrow T *^{1}(\Omega(\Gamma))\left(\cong T^{*} M\right),
$$

with rule of correspondence 


$$
\left(x_{i}, \partial_{t} x^{i}\right) \mapsto\left(\phi^{i}, \partial_{\mu} \phi^{i}\right),
$$

which produces the $i$ th - spinor field $\phi_{i}$ [14], where the action $\mathfrak{I}$, of the field $X$, infiltrates and transmits from particle to particle in the whole space $\Omega(\Gamma)$, using a configuration given by their Lagrangian $L$ (conscience operator), along all the trajectories of $\Omega(\Gamma)$. Then from a sum of trajectories $\int D_{F}(x(t))$, one has the sum $\int \mathrm{d}(\phi(x))$, on all the possible field configurations $C_{n, m}$. Extending these intentions to the whole space $\Omega(\Gamma) \subset \mathbb{M}$, on all the elections of possible paths whose statistical weight corresponds to the determined by the intention of the field, and realizing the integration in paths for an infinity of particles-fields in $T \Omega(\Gamma)$, it is had that

$$
\begin{aligned}
\mathfrak{I}\left(\phi^{i}(x)\right) & =\int_{T \Omega(\Gamma)} \omega(\phi(x)) \\
& =\lim _{\substack{N \rightarrow \infty \\
\delta s \rightarrow 0}} \frac{1}{B} \int_{-\infty}^{+\infty} \frac{\mathrm{d} \phi^{1}}{B} \cdots \int_{-\infty}^{+\infty} \frac{\mathrm{d} \phi^{n}}{B} \cdots \\
& =\prod_{i=1}^{\infty} \int_{-\infty}^{+\infty} \mathrm{e}^{\left.i \Im \phi^{i}, \partial_{\mu} \phi^{i}\right]} \mathrm{d} \phi^{i}(x(s)),
\end{aligned}
$$

where $B=\left[\frac{m}{2 \pi \hbar i \delta s}\right]^{1 / 2}$, is the amplitude of their

propagator and in the second integral of (14), we have expressed the Feynman integral using the form of volume $\omega(\phi(x))$, of the space of all the paths that are added in $T \Omega(\Gamma)$, to obtain the real path of the particle (where we have chosen quantized trajectories, that is to say, $\int \mathrm{d}(\phi(x))$,. Remember that the sum of all these paths is the interference amplitude between paths that are established under an action whose Lagrangian is $\omega(\phi(x))=\mathfrak{J}_{\xi(x)} \mathrm{d} \phi(x)$, where, if $\Omega(\mathbb{M})$, is a complex with $\mathbb{M}$, the space-time, and $C(\mathbb{M})$, is a complex or configuration space on $\mathbb{M}$, (interfered paths in the experiment given by multiple split $[11,15])$, endowed with a pairing

$$
\int: C(\mathbb{M}) \times \Omega *(\mathbb{M}) \rightarrow \mathbb{R},
$$

where $\Omega *(M)$, is some dual complex ("forms on configuration spaces"), i.e. such that "Stokes theorem" holds:

$$
\int_{\Omega \times C} \omega=\langle\mathfrak{I}, \mathrm{d} \omega\rangle,
$$

then the integrals given by (14) can be written as (to $m$ - border points and $n$-inner points as:

$$
\begin{aligned}
& \int_{\mathrm{T} \Omega(\Gamma)} \Im(\phi(x)) \mathrm{d} \phi(x) \\
& =\int_{\Omega\left(\Gamma_{t}\right) \times \cdots \times \Omega\left(\Gamma_{t^{m}}\right) \times \cdots} \mathfrak{J}_{q} \mathrm{~d} \phi_{1}^{m_{1}} \cdots \mathrm{d} \phi_{n}^{m_{n}} \cdots \\
& =\int_{\Omega\left(\Gamma_{t^{1}}\right)}\left(\int_{\Omega\left(\Gamma_{t^{2}}\right)} \cdots\left(\int_{\Omega\left(\Gamma_{t^{m}}\right)} \Im \mathrm{d} \phi_{1}^{m_{n}}\right) \cdots \mathrm{d} \phi_{n}^{m_{1}}\right) \cdots \text {, }
\end{aligned}
$$

This is an infiltration in the space-time by the direct action $\mathfrak{I}[4,6]$ that happens in the space $\Omega \times C$, to each component of the space $\Omega(\Gamma)$, through the expressed Lagrangian in this case by $\omega$, de (10) in (17), the integration of the space is realized with the infiltration of the time, integrating only spinor elements of the field.

Definition 1.2. (intentional action of $X$ ) [12]. Let $X$ be a field acting on the particles

$x_{1}(t), x_{2}(t), x_{3}(t), \cdots, \in \mathbb{M}$, and let $\mathfrak{I}$, be their action on the mentioned particles above, under an operator who recognizes the "target" in $\mathbb{M}$, (conscience operator). We say that $\mathfrak{I}$, is an conscientious intentional action (or simply intention) of the field $X$, if and only if

1) $\mathfrak{I}$, is the determination of the field $X$, to realise or execute (their force);

2) $\mathfrak{I}$, recognizes well their target, knowing what to do of the field $X$ (their direction $\Leftrightarrow$, follows a configuration patron)

The definition 1. 2., is summed establishing the principle:

Principle 2. [12,13]

Intention $=$ Determination of the field to realize a action + Knowledge of their objective

The knowledge of their target is the knowledge of their effect. This brings with it the cause - effect knowledge, which anticipates the action and determines their application.

But how to construct a conscience that integrates the time and space through the proper energy of the matter that constitutes it, and that could transform the matter constituted by these particles $x(s)$, what the intentional action wants?

The action must expand their intention, that is to say, must be transmitted from particle to particle "infecting" all space that it wants transform taking the same intention given by the action, using the relation of distance that is subjacent between connected virtual particles that take this intention (since these that are photons (bosons, fermions, gravitons, baryons, etc) shape the exchange of energy information between atoms and weave shaping the network of specific messages between the atoms).

Likewise, if $M$ is a space-time constituted by matter defined by the particles $x_{1}(t), x_{2}(t), \cdots$ and is desirable to realize a transformation of this matter transmitting an intention of an action $\mathfrak{J}$, given by a field $X$, through the states $\phi_{1}, \phi_{2}, \phi_{3}, \cdots$ such that it satisfies (17) with the angular relation between two connected states given by their angular momentum (and this way successively to all the remaining states) then the intention is transmitted like the quantum wave $\varphi$, of the state $\phi_{i}$, replaced with the state $\phi_{i},(i=1,2,3, \cdots)$ in the infinite homomorphism $[12,16]$ (which is of the type (14), later in the Section 4, we will talk more about it) 


$$
\varphi_{\phi_{1}}\left(t_{\phi_{1}^{\prime}}\right) \varphi_{\phi_{2}}\left(t_{\phi_{2}^{\prime}}\right) \varphi_{\phi_{3}}\left(t_{\phi_{3}^{\prime}}\right) \varphi_{\phi_{4}}\left(t_{\phi_{4}^{\prime}}\right) \cdots
$$

where the transmission of the quantum wave is realized on the spinor space of $t_{\sigma}$, where $t_{\sigma}$, is the nanotechnology that is wanted to create in the class $\sigma$. An interesting re-interpretation of (18) is when this quantum wave is a quantum code of intelligence spilled in the memory of a microprocessor for the achievement of an action of correction, re-composition, alignment, or restoration of a field of particles [7]. Here the distinguishable particles states are already bosons or fermions, they have a job or arrangement that eliminates an infinity of the states that by their sum of spins are annulled, leaving alone those who realize an effective action. They annul the shaken states or realize scattering [7].

Considering a space of configuration $C_{n, m}$, equivalent to the complex given for $C(\mathbb{M})$, composed for $n$-hypercubes $\mathrm{U}$, defined by $000 \cdots 0$-boxes, we can define a network of paths that will be able to establish routes of organized transformations on diagrams of Feynman type (with path integrals with actions given by $O_{c}$, and path integrals as given in (11)) which will establish the ideal route of the intention, considering the action in every node of the network. Then these arrangements can happen in the networks designed on a field of particles that can be arranged in $000 \cdots 0$ - boxes [16], where the action can be calculated in a point (node of the crystalline network of a field [17]) corresponding to the $n$-states of energy $\phi_{i} \quad(i=1,2,3, \cdots, n)$, superposed near the node for

$$
\begin{aligned}
& \oint[x]_{\mathrm{U}} \\
& =\int_{000 \cdots 0 \text {-boxes }} \mathrm{d} z^{n} \phi\left(X^{\alpha_{1}} W_{\alpha_{1}^{\prime}}\right) \phi\left(X^{\alpha_{2}} W_{\alpha_{2}^{\prime}}\right) \cdots \phi\left(X^{\alpha_{n}} W_{\alpha_{n}^{\prime}}\right),
\end{aligned}
$$

In the quantum zone, the quantum particles field is permanent and interminable, since matter and energy are equivalent and the atoms are interminable (they do not suffer wear), and what gets worn there are the linkages between atoms which can weaken or get lost for absence of a transmission of the states of suitable energy (routes given by path integrals). Nevertheless, infiltrating the intention on every path $\gamma$, and under the condition of permanent field given by the operators $O_{c}$, the transmission of the states will be able to turn revitalized by every node, transmitting the same information about every $000 \cdots 0$-box. We will call this characteristic a intentionality [12]. But every particle with regard to others takes their corresponding position, since they all have the same infiltrated intention, by which the synergic action is realized.

Principle 3. The nanotechnology is a science of the organization of nano-components of the matter, space or energy with quantum intention.

Example 1.1. The nano-components of different objects are the same, the only thing that changes is the arrangement of their atoms to form particles or molecules to create the different objects and realities in the space-time MI (see Figure 1).

Example 1.2. The intention of an action takes implicit the atomic visualization of every nano-component. Likewise, if $p_{1}, p_{2}, \cdots, p_{n} \in \mathbb{M}$, are $n$-particles with an finite arrangement of the atoms $a_{1}, a_{1}, \cdots, a_{m} \in \mathbb{S}_{\mathrm{NANO}}$, given by a structure $C_{\bullet}(\mathrm{MI})=C_{n, m}$, (configuration space in $M I)$, which considers configurations from $\mathbb{S}_{\text {NANO }}$, up to the particles of the material reality in $M T$, the intention from $s_{\mathrm{NANO}}$, generates the organised transformation in the space $M$. Likewise, we can say that the nano-space is the zone where there initiates an organized transformation of nano type (see Figure 2).

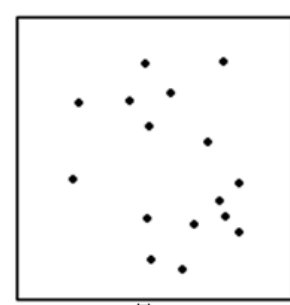

(I)

(a)

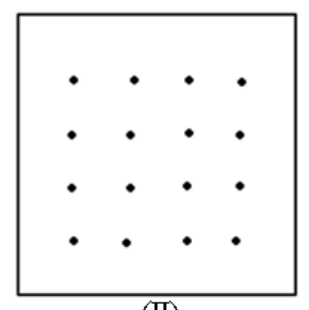

(II)

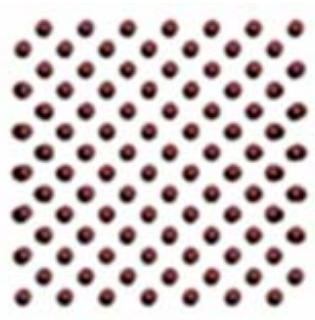

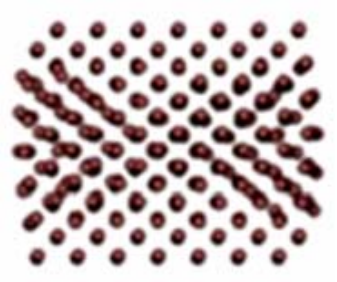

(b)

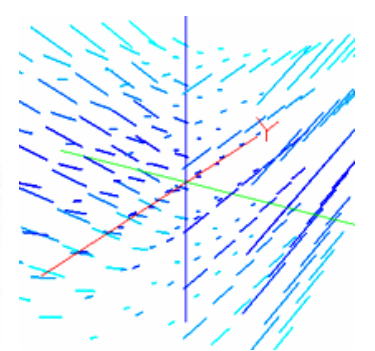

\section{.}

Figure 1. (a) Nano-components; (b) Field. (a) The preservation of the measure of the energy process in the nano-components is given for their invariance. The ensemble of the nano-components is realized without energy invariance. This demonstrates that the supported action in all nanotechnological process is the showed by the figure (I) remember that the object obtains their finished transformation in an established limit (Figure 3). (II) The action of the field is expanded to the whole space achieving that the showed configuration in (I), is transmitted to the whole space, object of the transformation in continuous form. This continuous transformation involves a homomorphism between the space of configuration and the space-time that characterizes the continuous map to the nanotechnological transformation. In the given simulating in (I), has been considered a field $X=\operatorname{VectorPlot} 3 D\left(2 x, 4 x, x^{\wedge} 2\right)$, whose expansion in $\mathbb{R}^{3}$, is the incise (II). The expansion preserve volume of matter, that is to say the volume of particles set that is transformed remains invariant. 


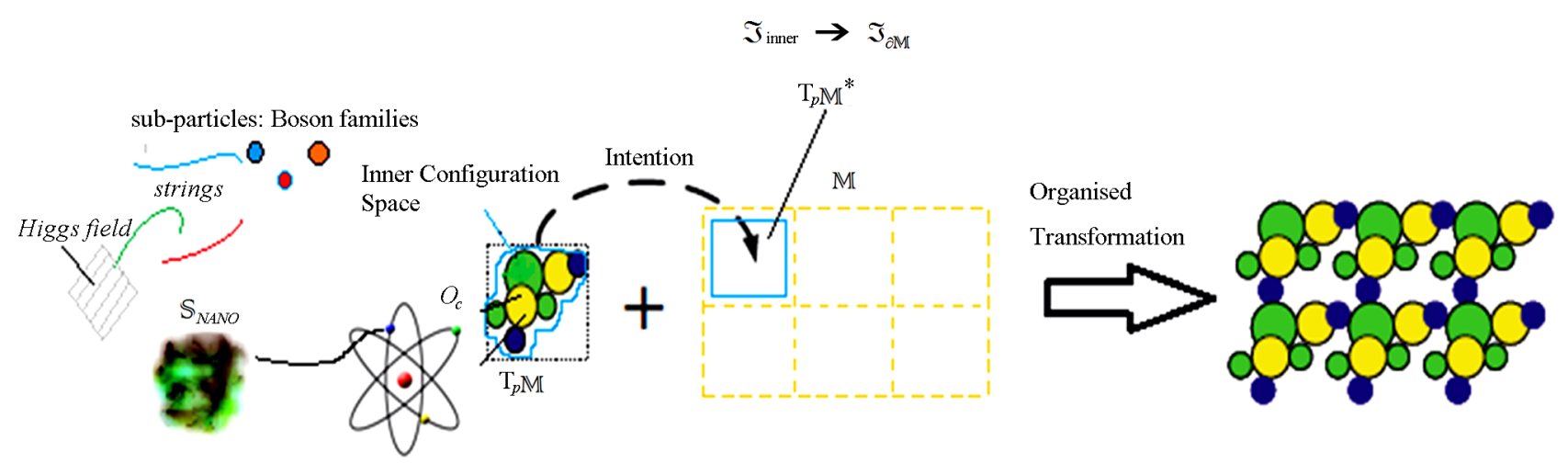

Figure 2. The intention infiltrated by the conscience given for $O_{c}$, establishes that the differential of the action $d \mathfrak{I}(\phi) h$ (using the energy (amplitude) that their propagator contributes $O_{F}$ ) can be visualised inside the configuration space through their boarder points (“targets" of the intention of the field $X$, and that happen in $\partial \mathrm{M}$ ), being also the interior points of the space $M$, intM, are the proper sources of the field (particles of the space $M$, that generate the field $X$ ). Then the intention of the field $X$, is the total action $\mathfrak{I}_{T}=\mathfrak{I}_{\partial M}\left(\mathfrak{I}_{\text {intm }}\right)$.

We consider the following postulate:

Postulate 1. Infinitely big is contained in infinitely small. It allows comprising the cause of any observable effect.

Due to the postulate 1, the measurement of a process of nanotechnology is realized according to their capacity of ensemble of the nano-components (particles) under an energy condition, that is to say, for every time $s$, of the particles $x(s)$, a map exists

$$
T_{t}: \mathcal{H} \rightarrow \mathcal{H}_{0},
$$

with rule of correspondence

$$
\phi(x) \mapsto T_{s}(\phi(x)),
$$

that for a sustainable period of time $T$, and considering their operator $O_{c}$, it had that the supported action satisfies

$$
\mathfrak{I}_{O_{c} T_{t}}=\lim _{T \rightarrow \infty}\left(\frac{1}{T}\right) \int_{0}^{T} O_{c}\left(T_{s} \phi\right) \mathrm{d} s,
$$

We will see eventually that a $\mu$, is always associated with a measure $\mu^{\wedge}$, on energy region space $J_{E} \subset \mathcal{H}$, with $\mu^{\wedge}\left(J_{E}\right)=1$, so that

$$
\mu\left(O_{c}\right)=\int_{J_{E}} O_{c}(\phi(s)) \mathrm{d} \mu^{\wedge}(\phi),
$$

Thus the limit given in (22) exists for each fixed $\phi$, and is independent of $\phi \in J_{E}$, then there is a measure $\mu$, on $J_{E}$, so that

$$
\lim _{T \rightarrow \infty}\left(\frac{1}{T}\right)^{T} \int_{0}^{T} O_{c}\left(T_{s} \phi\right) \mathrm{d} s=\int_{J_{E}} O_{c}(\phi) \mathrm{d} \mu(\phi),
$$

The measure $\mu$, has a very important property. Let $\mu$, be fixed and suppose $J_{E}$, the characteristic function of a measurable set $F \subset J_{E}$, then

$$
\begin{aligned}
& \left(\frac{1}{T}\right) \int_{0}^{T} \chi_{T^{-1}{ }_{u \nu v} F}(\phi) \mathrm{d} s \\
& =\left(\frac{1}{T}\right) \int_{0}^{T} \chi_{T_{u^{-1}} F}\left(T_{v} \phi\right) \mathrm{d} s \\
& =\left(\frac{1}{T}\right) \int_{0}^{T} \chi_{F}\left(T_{u} T_{v} \phi\right) \mathrm{d} s,
\end{aligned}
$$

so if the $\lim _{\mathrm{T} \rightarrow \infty}$, exists, then $\left(\mathrm{T}_{u}^{-1} F\right)=\mu(F)$, that is, the measure is invariant. Then we say that $T$, is measure preserving (see Figure 1).

The volume conservation of particles is the conservation of the flow of particles Flux $O_{c}$, in $\mathcal{H}$, then we have the following invariability principle of the measurement in any nanotechnology process:

Principle 4. In any nanotechnological process there is preserved the measurement of volume of the set of particles led by $O_{c}$, of the region of space-time that is transformed. There must not be losses of volume.

Example 1.3. In the Hamiltonian formulation of classical mechanics, the position and momentum of a system are described by the points of the cotangent bundle $\mathrm{T}^{*} \mathrm{M}$. Likewise, this is a symplectic manifold in a natural way. The physics aspects of the system are described by a so-called Hamiltonian function $H: \mathrm{T}^{*} \mathrm{M} \rightarrow \mathbb{R}$, and the evolution of the system is given by the flow of the Hamiltonian vector field $X_{H}$, associated to $H$, satisfying the Lioville integration theorem $[1,18]$.

In the results of this paper, this property will be discussed as fundamental in the nanotechnological chore.

\section{Intentionality}

We consider $\mathbb{M} \cong \mathbb{R}^{3} \times I_{t}$, the space-time of certain particles $x(s)$, in movement, and let $L$, be an operator that explains certain law of movement that governs 
the movement of the set of particles in $M$, of such way that the energy conservation law is applied for the total action of each one of their particles. The movement of all the particles of the space $M$, is given geometrically for their tangent vector bundle TM . Then the action due to $L$, on $\mathbb{M}$, is defined like [1]:

$$
\mathfrak{J}_{L}: \mathrm{TMI} \rightarrow \mathbb{R}
$$

with rule of correspondence

$$
\mathfrak{I}(x(s))=\operatorname{FluxL}(x(s)) x(s),
$$

and whose energy due to the movement is

$$
E=\mathfrak{I}-L,
$$

But this energy is given from their Lagrangian $L \in C^{\infty}$ ( TMI, $\left.\mathbb{R}\right)$, defined like [19]

$$
L(x(s), \dot{x}(s), s)=\mathrm{T}(x(s), \dot{x}(s), s)-\mathrm{V}(x(s), \dot{x}(s), s),
$$

If we want to calculate the action defined in (13) and (14), along a given path $\Gamma=x(s)$, we have that the action is

$$
\mathfrak{I}_{\Gamma}=\int_{\Gamma} L(x(s), x(s), s) \mathrm{d} s,
$$

If this action involves an intention (that is to say, is an intentional action) then the action is translated in all the possible field configurations, considering all the variations of the action along the fiber derivative defined by the Lagrangian $L$. Of this way, the conscience operator is the map

$$
O_{c}: \mathrm{TM} \rightarrow \mathrm{T}^{*} \mathrm{M},
$$

with corresponding rule

$$
O_{c}(v) w=\left.\frac{\mathrm{d}}{\mathrm{d} s} L(v+t w)\right|_{s=0},
$$

That is, $O_{c}(v) w$, is the derivative of $L$, along the fiber in direction $w$. In the case of $v=x^{\prime}(s)$, and $q=x(s), \quad \forall q \in \mathbb{M}, L(q, v)=E-V=1 / 2\langle v, v\rangle-V(q)$, we see that $O_{c}(v) w=\langle v, w\rangle$, so we recover the usual map $s^{b}: T M r \rightarrow T^{*} M$, (with $b$, Euclidean in $\mathbb{R}^{3}$ ) associated with the bilinear form $\langle$,$\rangle . Is here where the$ spin structure subjacent appears in the momentum of the particle $x(s)$.

As we can see, $\mathrm{T}^{*} \mathbb{M}$, carries a canonical symplectic form, which we call $\omega$. Using $O_{c}$, we obtain a closed two-form $\omega_{L}$, on TM, by setting [12]

$$
\omega_{L}=\left(O_{c}\right) * \omega
$$

Considering the local coordinates $\left(\phi_{i}, \partial_{\mu} \phi_{i}\right)$, to $\omega_{L}$, modeling the space-time $\mathbb{M}$, through $\mathcal{H}$-spaces, we have that (32) is

$$
\omega_{L}=\frac{\partial^{2} L}{\partial \phi^{i} \partial \partial_{\mu} \phi^{j}} \mathrm{~d} \phi^{i} \wedge \mathrm{d} \phi^{j}+\frac{\partial^{2} L}{\partial \phi^{i} \partial \partial_{\mu} \phi^{j}} \mathrm{~d} \phi^{i} \wedge \mathrm{d} \partial_{\mu} \phi^{j},
$$

Likewise, the variation of the action from the operator $O_{c}=\mathrm{d} \Im(\phi)=L\left(\phi, \partial_{\mu} \phi\right) \mathrm{d} \phi$, is translated in the differential

$$
\mathrm{d} \Im(\phi) h=\int_{\Gamma}\left(\frac{\partial L}{\partial \phi}-\frac{\mathrm{d}}{\mathrm{d} t} \frac{\partial L}{\partial \dot{\phi}}\right)(\phi(s), \dot{\phi}(s)) h(s) \mathrm{d} s,
$$

where $h(s): \Gamma \rightarrow$ TM, and is such that $\tau_{\mathrm{MI}} \circ h=\Gamma$, and $h\left(x_{1}\right)=h\left(x_{2}\right)=0$, to extreme points of $\Gamma$, $x\left(s_{1}\right)=q$, and $x\left(s_{2}\right)=q$. The total differential (34) is the symplectic form $\omega_{L}$, that constructs the application of the field intention expanding $2 n$-coordinates in (20). The space $\mathcal{X}^{1}(\Omega(\Gamma))$, is the space of differentiable vector fields on $\Omega(\Gamma)$, and $\Omega(\Gamma)$, is the manifold of trajectories (space-time of curves) that satisfies the variation principle given by the Lagrange equation that expresses the force $\mathrm{F}\left(x(s)_{j}\right), \quad(j=1,2, \cdots, n)$ generated by a field that creates one "conscience" of order given by their Lagrangian (to see the Figure 1(a)).

The operator $O_{c}$, is an operator that involves the Lagrangian but directing this Lagrangian in one specific fiber (direction) that is to say, prefixing that Lagrangian action in one direction. We remember that said map: $O_{c}: \mathrm{TM} \rightarrow \mathrm{T}^{*} \mathrm{M}, \quad$ have rule of correspondence $w \mapsto O_{c}(v) w$, where $w=L(v)$, with $L$, the classic Lagrangian. This defines the quantum conscience. If we locally restrict to $O_{c}$, that is to say, on the tangent space $\mathrm{T}_{x} \mathrm{M} \times \mathrm{T}_{x} \mathrm{M}, \quad \forall x \in \mathbb{M}(\cong \Omega(\Gamma))$, we have that [12]

$$
O_{c}: \mathrm{T}_{x} \mathrm{M} \times \mathrm{T}_{x} \mathrm{Mr}\left(\cong_{\text {locally }} \mathrm{TMI}\right) \rightarrow \mathrm{TM}^{*}
$$

with rule of correspondence

$$
(v, w) \rightarrow O_{c}(v) w,
$$

$O_{c}(v) w$, generalises the means of

$O_{c}(v) v=L(v), \forall v \in \mathrm{T}_{x} \mathrm{M}, \quad \forall x \in \Omega(\Gamma)$. Likewise, if $\mathfrak{I}: \mathrm{TM} \rightarrow \mathbb{R}$, with rule of correspondence $L(v) \mapsto \mathfrak{I}(L(v))=O_{c}(v) v$, then the total action along the trajectory $\Gamma$, will be

$$
\mathfrak{I}_{\Gamma}=\int_{\Omega(\Gamma)} O_{c}(v) v=\int_{\Omega(\Gamma)} L(v),
$$

that is the integral given in (16).

In the forms language, the conscience operator comes given by the map $\omega_{L}: \mathrm{TM} \rightarrow \mathrm{T}^{*} \mathrm{M}$, with rule of correspondence given by (32). The quantum conscience shape a continuous flux of energy with an intention, involving a smooth map $\pi$, (defined in the example 1). Then the conscience operator is related with the action $\mathfrak{I}$, and the trajectories $\gamma_{s}$, through of the following diagram [12]:

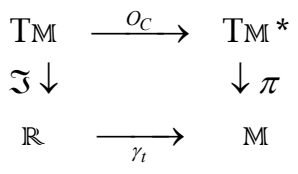


Proposition 2.1. The diagram (37) is commutative.

Proof. By construction and nature of the operator $O_{c}$.

Consider the following basic properties of the operators $O_{c}$. Let $\mathcal{O}\left(x-x^{\prime}\right)$ [20] defined in the footnote 1, and $\phi(x) \in \mathcal{H}$, where the space $\mathcal{H}$, is the set of points

$$
\mathcal{H}=\left\{\phi(x) \in[m] \mid[m] \subset \mathrm{T}^{*} \mathbb{M}\right\}^{1}[3],
$$

Points of phase space are called states of the particle system acting in the cotangent space of Mr. Thus, to give the state of a system, one must specify their configuration and momentum.

Example 2.1 [12]. Let $\pi: \mathrm{T}^{*} \mathrm{M} \rightarrow \mathrm{M}$, be (like given by commutative diagram) and $\gamma: \mathbb{R} \rightarrow \mathrm{T} C_{n, m}$, then $\pi \circ \gamma: \mathbb{R} \rightarrow \mathbb{M}$, describes the curve in the configuration space, which also describes the sequence of configuretions through which the particles system passes to different strata of co-dimension one (see Figure 1). Every strata correspond to a phase space of $m$, particles that are moved by curve $\gamma$, and directed from their energy states $\mathrm{d} \phi(x)$, by $\pi$, to $n$, particles $\phi(x)$.

This defines our intentional conscience. Then are true the following properties:

1) $\mathcal{O}\left(x-x^{\prime}\right) \phi(x)=\delta\left(x-x^{\prime}\right) \phi(x), \forall x, x^{\prime} \in \mathbb{M},{ }^{2}$

2) $\quad O_{c}\left(x^{\prime}\right) \phi\left(x^{\prime}(s)\right)=\mathcal{O}\left(x-x^{\prime}\right), \forall x, x^{\prime} \in \mathbb{M}$, and $s \leq t$

3) $\int_{\mathcal{H}} O_{c}(\phi(s)) \mathrm{d} \phi=\mathfrak{I}_{O_{c}}$;

$\mathrm{d} \mathfrak{I}_{O_{c}}(\phi(s)) / \mathrm{d} \phi=O_{c}(x(s))$, in the unlimited space $\mathcal{H}$,

4) $O_{c}=\delta\left(s-s^{\prime}\right)$, if and only if $\delta x(s) / \delta x\left(s^{\prime}\right)=\delta\left(s-s^{\prime}\right), \forall s \leq t$, then $\mathrm{F}(x(s))=x(s)$,

5) $\mathcal{O}^{-1}\left(x-x^{\prime}\right) O_{c}(x(s))=-\Delta_{F}\left(x-x^{\prime}\right) \delta\left(x-x^{\prime}\right)$,

$\forall x, x^{\prime} \in \mathbb{M}$, and $s \leq t$,

6) $\int_{\mathcal{H}} O_{c}(\phi(s)) \mathrm{d} \phi=\int_{\Omega} \mathcal{O}\left(x-x^{\prime}\right) x(s) \mathrm{d}(x(s))$.

All properties are demonstrated in [12], the reader cans to find all details in this reference.

\footnotetext{
${ }^{1}$ The corresponding cotangent space to vector fields is:

$\mathrm{T}^{*} \mathcal{X}^{1}(\mathrm{M})=\left\{\left(\phi, \partial_{\mu} \phi\right) \in \mathcal{H} \times \mathrm{T} \mathcal{X}^{1}(\mathrm{M}) \mid \partial_{\mu} \phi=\nabla_{\mu} \xi, \forall \xi \in \mathcal{X}^{1}(\mathrm{M})\right\}$.
}

Here $[m]=\mathrm{T}^{*} C_{n, m}$.

${ }^{2}$ In the general sense the functional derivative $\frac{\delta \varphi_{a}(y)}{\delta \varphi_{b}(x)}=\delta_{b a} \delta^{n}(y-x)$, implies

$$
\delta \varphi_{b}(y)=\sum_{a} \int \delta^{n}(y-x) \delta \varphi_{a}(x) \delta_{b a} \mathrm{~d} x,
$$

but does not imply

$$
\delta \varphi_{b}(y)=\delta_{b a} \delta^{n}(y-x) \delta \varphi_{a}(x) .
$$

Now we consider the space-time $M$, like space where $\mathbb{R}^{d} \times I_{t}, d=3$, is the macroscopic component of the space-time and we called $\mathbb{F}$, the microscopic component of the space-time of ratio $10^{-33} \mathrm{~cm}$ (length of a string [21]). For previously described the quantum zone of the space-time $\mathcal{M}$, is connected with $\mathcal{N}$, which we will called virtual zone of the space-time (zone of the space-time where the process and transformation of the virtual particles happens) are connected by possibilities causal space generated by certain class of photons and by the material particles interacting in the material space time, with permanent energy and the material particles re-combining their states they become in waves on having moved in $\mathbb{R}^{d} \times I_{t}$, on any Feynman path. Likewise we can define the space of this double fibration of quantum processing as [12]:

$$
\begin{aligned}
\mathcal{L}=\{ & O_{c}\left(\phi, \partial_{\mu} \phi, x(t), t\right) \in C^{2}\left(\mathbb{R}^{d} \times I_{t}\right) \mid \frac{\partial^{2}}{\partial t^{2}} \\
& \left.-\nabla^{2}\left(O_{c}\left(\phi, \partial_{\mu} \phi, x(t), t\right)\right)=0\right\},
\end{aligned}
$$

with the states $\phi$, of quantum field are in the quantum zone $\mathcal{M}$. Let $\mathcal{N}$, the ambi-space (set of connection and field) defined as:

$$
\mathcal{N}=\left\{(X, \nabla) \in \mathscr{M} \times \mathcal{L} \mid \nabla_{x^{\prime} y^{\prime}}^{x y} \Psi+\Phi(X)=0\right\},
$$

where $\nabla$, is the connection of virtual field $X$, with the quantum field $Y$, and $\Psi$, is the field whose action is always present to create perceptions in the quantum zone connected with $\Phi$ (2-form) [22]. Then we can create the correspondence given by the double fibration [16]:

(space of processing of quantum particles)

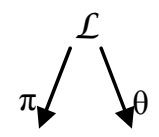

(virtual zone of $\mathcal{M}$ (quantum zone the space-time) $\mathcal{N} \quad$ of the space-time)

This double fibration conforms the interrelation between $\mathcal{M}$, and $\mathcal{N} . \forall x(t) \in \mathcal{M}$, give beginning to a complex submanifold (that represents the spaces where are the quantum hologram) that includes all these quantum images given by quantum holograms, why? Because these complex submanifolds, considering the causal structure given in the space-time by the light cones [23], of all trajectories that follows a particle in the space-time [16], they can write using (41) as:

$$
\Theta_{x}=\theta\left[\pi^{-1}(x)\right],
$$

of $\mathcal{N}$, such that $\Theta_{x}=\mathbb{P}^{1} \times \mathbb{P}^{1}$, which by space-time 
properties to quantum level represents the space of all light rays that transit through $x$, conforming a hypersurface (projective surface) that is a light surface. This surface is called the sky $x$ [24]. A sky in this context represents the set of light rays through $x$ (bosons) that comes of the virtual field.

If $\mathcal{M} \cong \mathbb{C}^{4}$, then $\mathbb{M r}=\mathcal{M} \times Q_{x}$, is the complete universe (include the super-symmetries [25]). But, what is there of our quantum universe with regard to our real universe (included the material part given by the atoms)?

The answer is the same, we have an universe of ten dimensions and $\mathbb{M}=\mathcal{N} \times \mathcal{M}$, where the quantum representation of the object $x(s)$, is the quantum space-time $\mathcal{M}=\mathbb{R}^{3} \times I_{t}$ (which is the space-time as the Einstein perception) then the images of the virtual particles are $\mathrm{C}^{2} \times Q_{x},[11]$, then the execution operator $\mathfrak{I}$, that proceeds to connect virtual particles through the paths which have path integrals on a double fibration, establishing the material-quantum-virtual connection required to a total reality:
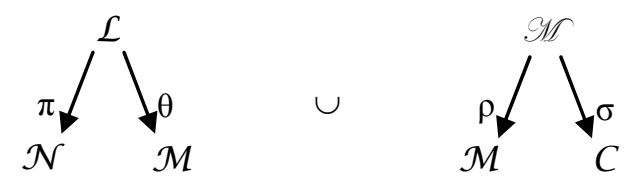

where $C$, is the material part connected with the quantum zone of the space-time (space taken by atoms) $\mathcal{M}$. The corresponding path integral that connects virtual particles in the whole fibration is the integral of line type (5) defining feedback connection:

$$
\mathfrak{J}\left(\mathfrak{J}_{Q_{x}}(x(s))\right)=\oint_{\Gamma} O_{c}\left(\theta\left(\pi^{-1}\left(\sigma\left(\rho^{-1}(x)\right)\right)\right)\right) \mu_{s},
$$

always with the space $\left\{x(s) \in \mathcal{M} \mid \Theta_{x} \subset \mathcal{N}\right\}$, to the permanent field actions. Then the reality state is the obtained through the integral of perception (44), considering the fibre of the corresponding reality in the argument of the operator $O_{c}$, of the integrating from (44).

Proposition 2.2. All transformation of a reality includes bosons of the $\mathcal{N}$ - field.

Proof. To demonstrate this, is necessary to prove that the cohomological group on $\mathcal{N}$, is the same cohomological group modulus a seated class in $\mathcal{N}$, of the material reality and the corresponding for the quantum reality. For it result useful the stacks concept in physics, where is possible to tack bosons to construct superior physical spaces using bosons branes (see corollary of [26]). On the other hand, any open numerable covering of bosons in $\mathcal{N}$, contains a finite subcovering in $\mathcal{M}$, which is guaranteed by the sky $Q_{x}$. But a sky in this context represents the set of light rays through $x$ (bosons) that comes of the virtual field.
In a plane of reality of the space $\mathcal{N}$, we can establish a commutative diagram similar to the given in (37), considering fibers of the topological space $s k y Q_{x}$, that at all times do that the integral submanifolds in $I L$ (through of the double fibrations of (59)) connect both realities determined in $\mathcal{N}$, and $\mathcal{M}$, along these submanifolds:

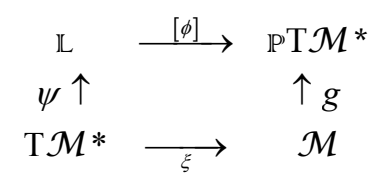

where $\mathbb{L}=\left\{\psi \mid \psi\left(\theta\left(\pi^{-1}(\phi)\right)\right)=0\right\}$, with

$\left.\mathbb{L} \rightarrow \mathbb{P}^{\mathrm{T}} \mathcal{M}^{*}\right|_{\pi^{-1}(\phi)}=O(1,1)$, where $O(1,1)$, is a homogeneous bundle of lines due to that the sky $Q_{x} \cong \mathbb{P}^{1} \times \mathbb{P}^{1}$, since the normal bundle $\mathrm{N} \rightarrow Q_{x}$, in every sky $Q_{x}$; is isomorphic to $J^{1} O(1,1)$. In particular there is an exact sucession

$$
0 \rightarrow \Omega^{1} \otimes O(1,1) \rightarrow \mathrm{N} \rightarrow O(1,1) \rightarrow 0,
$$

which allows to have a composition of the reality in $\mathcal{M}$, through fields that come of $\mathcal{N}$. Then the quantum virtual composition of both realities is given by the moduli stack:

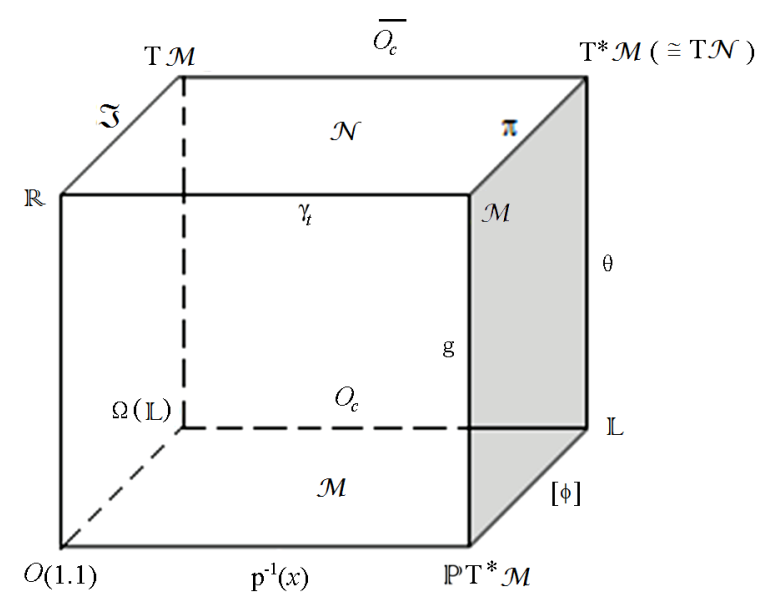

\section{Intention Transmission and Introspection}

Considering the quantizations of our Lagrangian system describe in (17), (31) and (32) on $\mathbb{R}^{n}, n \geq 2$, coordinated by $\left\{x^{j}\right\}$, we describe terms of a graded commutative $C^{\infty}(\mathrm{M})$ - algebra $H$, with generating elements

$$
\left\{\partial_{\mu} x^{a}, \partial_{\mu} x_{\lambda}^{a}, \partial_{\mu} x_{\lambda_{1} \lambda_{2}}^{a}, \cdots, \partial_{\mu} x_{\lambda_{1} \lambda_{2} \cdots \lambda_{k}}^{a}, \cdots\right\},
$$

and the bi-graded differential algebra $H^{*}$, of differential forms (the Chevalley-Eilenberg differential calculus

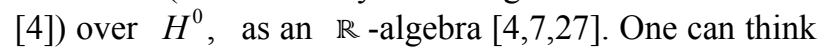


of generating elements (44) of $H$, as being sui generis coordinates of even and odd fields and their partial derivatives. The graded commutative $\mathbb{R}$-algebra $H^{0}$, is provided with the even graded derivations (called total derivatives)

$$
d_{\lambda}=\partial_{\lambda}+\sum_{0 \leq 1 \Lambda \mid} \partial_{\lambda+\Lambda}^{a} \partial_{a}^{\Lambda}, \quad d_{\Lambda}=d_{\lambda_{1}} \cdots d_{\lambda_{k}}
$$

where $\Lambda=\left(\lambda_{1} \cdots \lambda_{k}\right),|\Lambda|=k$, and $\lambda+\Lambda=\left(\lambda, \lambda_{1} \cdots \lambda_{k}\right)$, are symmetric multi-indices. One can think of even elements

$$
\begin{aligned}
L & =\mathcal{L}\left(x^{\lambda}, \partial_{\mu} x^{a}\right) \mathrm{d}^{n} x, \\
\delta L & =\mathrm{d} \partial_{\mu} x^{a} \wedge \mathcal{E}_{a} \mathrm{~d}^{n} x \\
& =\sum_{0 \leq|\Lambda|}(-1)^{|\Lambda|} \mathrm{d} \partial_{\mu} x^{a} \wedge \mathrm{d}_{\Lambda}\left(\partial_{q}^{\Lambda} L\right) \mathrm{d}^{n} x,
\end{aligned}
$$

where we observe that $\delta L$, is the 2 -form given by $\omega_{L}$, in the formula (33) with $n=2$, and $\Lambda=\lambda_{1} \lambda_{2}$.

Now we consider the dual part of the space $(H, \Omega(\Gamma))$, that is to say, the space $\left(H^{*}, L\right)$. We consider quantize this Lagrangian system in the framework of perturbative Euclidean QFT. We suppose that $L$, is a Lagrangian of Euclidean fields on $\Omega(\Gamma) \subseteq \mathbb{R}^{n}$. The key point is the algebra of Euclidean quantum fields $B_{\Phi}$, given as $H^{0}$, the graded commutative. It is generated by elements $\phi_{a}^{\lambda \Lambda}(x), \forall x \in \Omega(\Gamma)$. For any $x \in \Omega(\Gamma)$, there is a homomorphism belonging to the space $H \rightarrow \operatorname{Hom}(\mathrm{T}(H), D)$, (with homomorphisms $H \rightarrow \operatorname{Hom}(\mathrm{T}(H), D)$, given for $D G$-algebra of cycles):

$$
\begin{aligned}
& \gamma_{x}: \mathfrak{J}_{a_{1} \cdots a_{r}}^{\Lambda_{1} \cdots \Lambda_{r}} \partial x_{\Lambda_{1}}^{a_{1}} \cdots \partial x_{\Lambda_{r}}^{a_{r}} \\
& \mapsto \mathfrak{J}_{a_{1} \cdots a_{r}}^{\Lambda_{1} \cdots \Lambda_{r}}(x) \phi_{x \Lambda_{1}}^{a_{1}} \cdots \phi_{x \Lambda_{r}}^{a_{r}}, \mathfrak{J}_{a_{1} \cdots a_{r}}^{\Lambda_{1} \cdots \Lambda_{r}} \in \mathrm{C}^{\infty}(\Omega(\Gamma))
\end{aligned}
$$

of the algebra $H^{0}$, of classical fields to the algebra $B_{\Phi}$, which sends the basic elements $\partial x^{a}{ }_{\Lambda} \in H^{0}$, to the elements $\phi_{a}^{\lambda \Lambda} \in B_{\Phi}$, and replaces coefficient functions $\mathfrak{I}$, of elements of $H^{0}$, with their values $\mathfrak{I}(x)$ (executions) at a point $x$. Then a state $\langle$,$\rangle of B_{\Phi}$, is given by symbolic functional integrals

$$
\begin{aligned}
& \left\langle\phi_{x_{1}}^{a_{1}} \cdots \phi_{x_{k}}^{a_{k}}\right\rangle \frac{1}{N} \int_{\mathcal{H}} \phi_{x_{1}}^{a_{1}} \cdots \phi_{x_{k}}^{a_{k}} \\
& \times \exp \left\{-\int_{\Omega(\Gamma)} O_{c}\left(\phi_{x \Lambda}^{a}\right) \mathrm{d}^{n} x\right\} \prod_{x}\left[\mathrm{~d} \phi_{x}^{a}\right],
\end{aligned}
$$

where this is an integral of type $\int_{\mathcal{H} \times \Omega(\Gamma)} O_{c}(x(s)) \mathrm{d} \phi$, as was given by the properties. Then when the intention expands to the whole space, infiltrating their information on the tangent spaces images (map (35)) of the cotangent bundle $\mathrm{T}^{*} \mathrm{M}$, (given by the imagen of $\phi(x)$, under $\left.\mathrm{d} \Im_{O_{c}}\right)$ then their intentionality will be the property of the field to spill or infiltrate their intention from a nano level of strings inside the quantum particles. Then from the energy states of the particles, and considering the intention spilled in them given by $O_{c}(\phi)$, we have the homomorphism (50) that establishes the action from $\mathbb{M} \cong(\Omega(\Gamma))$, to $\partial M \mathbb{M}$, for their transformation through the action $\mathfrak{I}_{\mathrm{T}}$, defined in (28) to any derivation given through their conscience operator (fiber (31)), like the graded derivation $\tilde{\partial}$, (considering the derivatives $\left.O_{c}(x)=\left(O_{c}\right) * \omega, \quad D_{F}=\mathrm{d} \phi\right)$ :

$$
\begin{aligned}
& \tilde{\partial}: \phi_{x \Lambda}^{a} \mapsto\left(x, \partial_{\mu}^{a} x\right) \mapsto\left(O_{c}(x), \omega\right) \\
& \mapsto\left(O_{c}^{*}(x), \omega_{L}\right)=O_{c}^{*}\left(\phi_{x \Lambda}^{a}\right) \omega,
\end{aligned}
$$

of the algebra of quantum fields $B_{\Phi}$,. With an odd parameter $\alpha$, let us consider the automorphism

$$
\hat{U}=\exp \{\alpha \tilde{\partial}\}=I d+\alpha \tilde{\partial},
$$

of the algebra $B_{\Phi}$, This automorphism yields a new state $\langle$,$\rangle , of B_{\Phi}$, given by the equality

$$
\begin{aligned}
& \left\langle\phi_{x_{1}}^{a_{1}} \cdots \phi_{x_{k}}^{a_{k}}\right\rangle \frac{1}{N^{\prime}} \int_{\mathcal{H}} \hat{U}\left(\phi_{x_{1}}^{a_{1}}\right) \cdots \hat{U}\left(\phi_{x_{k}}^{a_{k}}\right) \\
& \times \exp \left\{-\int_{\Omega(\Gamma)} O_{c}\left(\hat{U}\left(\phi_{x \Lambda}^{a}\right) \mathrm{d}^{n} x\right\} \prod_{x}\left[\mathrm{~d} \hat{U}\left(\phi_{x}^{a}\right)\right],\right.
\end{aligned}
$$

where the energy state has survived, since $\mathrm{d} \hat{U}\left(\phi_{x}^{a}\right)=\mathrm{d} \phi_{x}^{a}$. That because the intention is the same. The intention has not changed.

What happens towards the interior of every particle? What is the field intention mechanism inside every particle?

To answer these questions we have to internalise the actions of field $X$, on the particles of the space $M$, and consider their spin. But for it, it is necessary to do the immersion of the Lagrangian $\omega$, defined as the map

$$
\omega: \mathrm{L} \rightarrow \mathrm{M}^{2 n}, 3
$$

with rule of correspondence

$$
Z_{i} \mapsto \omega\left(Z_{i}\right),
$$

where the image of the 1-form $\omega$, that the Lagrangian defines, $\omega\left(Z_{i}\right)$, is a symplectic form [1], and the variable $Z_{i}$, is constructed through the algebraic equations $W^{a}\left(Z_{i}\right)=0$ [28]. They describe the $k$-dimensional hypersurfaces denoted by $S$, such that $S \subset \mathcal{H}$, where

\footnotetext{
${ }^{3}$ Having chosen $M^{2 n}$, is to consider the two components of any point in the space $\mathbb{C}^{N}$, (that we are considering isomorphic to the ambient space of any quantum particle $x(s)$, in the space-time) to have the two components that characterise any quantum particle $x(s)$, that is their spin (direction) and their energy state (density of energy or "living force of the particle"). $\mathbb{L}$, is the corresponding Lagrangian submanifold of the symplectic structure given by $\left(M^{2 n}, \omega\right)$.
} 
$\mathcal{H}$, is the phase space defined in the Section 2. The in$\operatorname{dex} a=1, \cdots, q$, runs over the number of polynomials $W^{a}\left(Z_{i}\right)$, in the variables $Z_{i}$, and $i$, runs over the dimension of the ambient manifold which is assumed to be $\mathbb{C}^{N}$. If the space is a complete intersection, the constraints $W^{a}\left(Z_{i}\right)$, (there is exact solution to $\left.W^{a}\left(Z_{i}\right)=0\right)$, are linearly independent and the differential form

$$
\Theta^{(n-k)}=\in_{a_{1} \cdots a_{N-k}} \mathrm{~d} W^{a_{1}} \wedge \cdots \wedge \mathrm{d} W^{a_{N-K}},
$$

is not vanishing. In this case, $q=N-k$, and the dimension of the surface is easily determined. For example, if the hypersurface is described by a single algebraic equation $W(Z)=0$, the form (60) is given by $\Theta^{(1)}=\mathrm{d} W$. On the other hand, if the hypersurface is not a complete intersection, then there exists a differential form

$$
\Theta^{(n-k)}=T_{A\left[a_{1} \cdots a_{N-k}\right]} \mathrm{d} W^{a_{1}} \wedge \cdots \wedge \mathrm{d} W^{a_{N-K}} \wedge \eta^{A,(N-k-q),}
$$

where $\eta^{A,(N-k-q)}$, is a set of $N-k-q$, forms defined such that $\Theta^{(N-k)}$, is non-vanishing on the constraints $W^{a}\left(Z_{i}\right)=0$, and $T_{A\left[a_{1} \cdots a_{N-k}\right]}$, is a numerical tensor which is anti-symmetric in the indices $a_{1} \cdots a_{q}$. The construction of $\eta^{A,(N-k-q)}$, depends upon the precise form of the algebraic manifold (variety of the equations

$\left.W^{a}\left(Z_{i}\right)=0\right)$. In some cases a general form can be given, but in general it is not easy to find it and we did not find a general procedure for that computation.

To construct a global form on the space $S$, one can use a modification of the Griffiths residue method [28], by observing that given the global holomorphic form on the ambient space

$\Omega^{(N)}=\epsilon^{i_{1} \cdots i_{N}} \mathrm{~d} Z_{i_{1}} \wedge \cdots \wedge \mathrm{d} Z_{i_{N}}$, we can decompose the $\left\{Z_{i}\right\}$ 's, into a set of coordinates $\mathrm{Y}^{a}=W^{a}(Z)$, and the rest. By using the contraction with respect to $q$, vectors $\left\{\underline{Z}_{i}^{a}\right\}$, the top form for $S$, can be written as

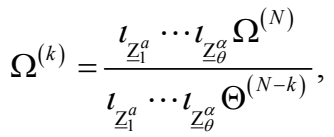

which is independent from $\left\{\underline{Z}_{i}^{a}\right\}$, as can be easily proved by using the constraints $W^{a}\left(Z_{i}\right)=0$. Notice that this form is nowhere-vanishing and non singular only to the case of $C Y$ - space (Calabi-Yau manifold). The Calabi-Yau manifold is a spin manifold and their existence in our space $\mathrm{M}^{2 n}$, like product of this construction is the first evidence that a spin manifold is the spin of our space-time due to their holomorphicity [29]. The vectors $\left\{\underline{Z}_{i}^{a}\right\}$, play the role of gauge fixing parameters needed to choose a polarisation of the space $S$, into the ambient space.

For example, in the case of pure spinor we have: the ambient form $\Omega^{(16)}=\epsilon_{\alpha_{1} \cdots \alpha_{6}} \mathrm{~d} \lambda_{i_{1}} \wedge \cdots \wedge \mathrm{d} \lambda_{i_{6}}, \quad$ and
$\Theta^{(5)}=\lambda \gamma^{m} \mathrm{~d} \lambda \lambda \gamma^{m} \mathrm{~d} \lambda \lambda \gamma^{m} \mathrm{~d} \lambda \mathrm{d} \lambda \gamma_{m n p} \mathrm{~d} \lambda$. From these data, we can get the holomorphic top form $\Omega^{(11)}$, introducing five independent parameters $\underline{\lambda}$, and using the formula (56).

The latter is independent from the choice of parameters $\underline{\lambda}$, (however, some care has to be devoted to the choice of the contour of integration and of the integrand: in the minimal formalism, the presence of delta function $\delta(\lambda)$, might introduce some singularities which prevent from proving the independence from $\underline{\lambda}$, as was pointed out in [30,31]). Using $\Omega^{(k)} \wedge \Omega^{(k)}$, one can compute the correlation functions by integrating globally defined functions. When the space is Calabi-Yau, also exists a globally-defined nowhere vanishing holomorphic form $\Omega_{h o l}{ }^{(k \mid 0)}$, such that $\Omega_{h o l}{ }^{(k \mid 0)} \wedge \Omega_{h o l}{ }_{(k \mid 0)}$, is proportional to $\Omega^{(k)} \wedge \underline{\Omega}^{(k)}$. The ratio of the two top forms is a globally defined function on the $C Y$ - space. In the case of the holomorphic measure $\Omega_{h o l}{ }^{(k \mid 0)}$, the integration of holomorphic functions is related to the definition of a contour $\gamma \in S$, in the complex space

$$
\left\langle\prod_{A} \mathcal{O}\left(Z_{i}, p_{A}\right)\right\rangle \int_{\gamma \in S} \Omega^{(k, 0)} \prod_{A} \mathcal{O}_{0}\left(Z_{i}, p_{A}\right)
$$

where $\mathcal{O}\left(Z_{i}, p_{A}\right)$, are the vertex operators of the theory localized at the points $p_{A}$, of the Riemann surface and $\mathcal{O}_{0}\left(Z_{i}, p_{A}\right)$, is the zero-mode component of the vertex operators. Newly our conscience operator come given by the form $\Omega^{(k, 0)}$.

Example 3.1. All Calabi-Yau manifolds are spin. In hypothetical quantum process (from point of QFT view), to obtain a Calabi-Yau manifold is necessary add (or sum) strings in all directions. In the inverse imaginary process, all these strings define a direction or spin. The strings themselves are Lagrangian submanifolds whose Lagrangian action is a path integral.

Let Mr, be a complex orientable Riemannian manifold (manifold that we consider as model of the space of the material and quantum reality) and $G$, the topological subjacent group to the manifold. Let $s \subset T^{*} \mathbb{M}$, be a conic and regular involutive submanifold.

The causality of all particle $p \in \mathbb{M}$, in the space $s$, is determined by the causality in the cotangent space $\mathrm{T} * \mathrm{M}$, to know

$$
\mathfrak{C}=\left\{C_{p} \mid C_{p} \subset \mathrm{T}_{p}^{*}(\mathbb{M}), \forall p \in \mathbb{M}\right\} \quad \text { (see Figure 3) }
$$

where $C_{p}$, is the corresponding light cone of the particle $p$, where all universe line is $\gamma_{t} \subset C_{p} \forall p \in \mathbb{S}$, with $\mathbb{s}$, is a microscopic space of $\mathbb{M}$. Given that this microscopic space have a structure of cause-effect induced for their proper causal structure $\mathfrak{C}$, then by the postulate 1 , we will refer to the above mentioned space like nano-space.

In a nano-space there is a structure of Hilbert space, since this has a pre-hilbert structure represented as a 


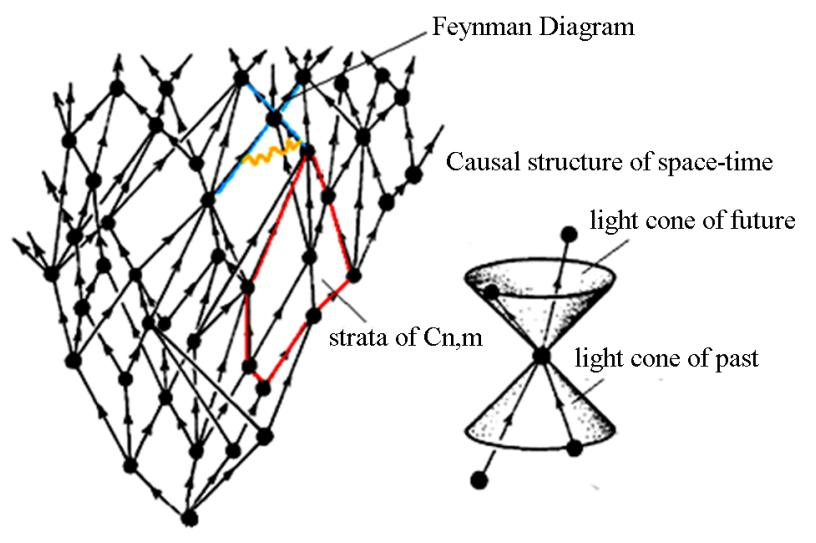

Figure 3. Causal structure and tree of the strata.

structure of interior product "(,)" which generates a Hilbert space through the quadratic form of their endomorphisms [32]. The space spin or spinor is a fibre of a bundle spinor in the orientable complex Riemanniana manifold $M$ [33].

We write $S(E)$, for the space defined as

$$
S(E)=\left\{\varphi \mid \varphi(v)^{2}=-(v, v) I d, \forall v \in E\right\},
$$

If $n(=\operatorname{dim} E)$, is odd then let $\varphi$, denote one of $\varphi^{ \pm}$. Recall that their corresponding algebra

$\mathfrak{s o}(E)=\{X \in \operatorname{End}(S) \mid(X v, w)=-(v, X w), \forall v, w \in E\}$,

If $v, w \in E$, are such that $(v, w)=0$, then let $X(v, w) \in \operatorname{End}(V)$, be defined by

$$
X(v, v) u=(u, w) v-(u, v) w, \forall u \in V,
$$

Then $X(u, v) \in \mathfrak{s o}(E)$. Let $e_{1}, \cdots, e_{n}$, be an orthonormal basis of $V$. Set $X_{i j}=X\left(e_{i}, e_{j}\right)$, for $i<j$. Then $X_{i j}, i<j$, is a basis for $\mathfrak{s o}(E)$. We define a linear map $\mu$, of $\mathfrak{s o}(E)$, into $\operatorname{End}(S(E))$, by

$$
\mu\left(X_{i j}\right)=-(1 / 2) \gamma\left(e_{i}\right) \gamma\left(e_{j}\right),
$$

A direct calculation shows that

$$
[\mu(X), \mu(Y)]=\mu[X, Y], X, Y \in \mathfrak{s o}(E),
$$

This implies that $(\mu, S(E))$, defines a module for $\mathfrak{s o}(E)$. Dicho modulo es el espacio $\mathfrak{s}_{N A N O}$.

Proposition 3.1. If $n$, is odd then up to equivalence $(\mu, S(E))$, is independent of the choice of $\gamma^{ \pm}$, and $(\mu, S(E))$, is irreducible. If $n$, is even then we set $S^{+}(V)=\wedge^{e v} W, \quad S^{-}(V)=\wedge^{\text {odd }} W$. Then $S^{ \pm}(V), \quad$ is invariant under $\mu$, and each defines an irreducible representation of $\mathfrak{s o}(V)$.

Proof. If $n$, is odd, set $V^{\prime}=\left\{v \in V \mid\left(v, v_{0}\right)=0\right\}$. Then our construction implies that $S(V)=S\left(V^{\prime}\right)$. If $v \in V^{\prime}$, set $\gamma^{\prime}(v)=\gamma\left(v_{0}\right) \gamma(v)$. Then $\gamma^{\prime}(v)^{2}=-(v, v) I$. Let $S^{\prime}$, be a non-zero subspace of
$S(V)$, of minimal dimension that is invariant under $\gamma^{\prime}\left(V^{\prime}\right)$. Then $S^{\prime}$, satisfies the definition of spinor space. If $n$, is odd then up to isomorphism there are exactly two spaces of spinors and they are each of dimension $2^{n / 2}$. In the case of even dimension, only there is one space of spinor under isomorphism of the dimension also $2^{n / 2}$. Then $\operatorname{dim} S^{\prime}=\operatorname{dim} S(V)$. Hence $S^{\prime}=S(V)$. So $\mu$, is irreducible in this case.

We now relabeling the orthonormal basis that we are using. If $n=2 k+1$, let $e_{0}=v_{0}, e_{i}, \cdots, e_{2 k}$, be an orthonormal basis of $V$. If $n=2 k$, then take $e_{1}, \cdots, e_{2 k}$. We assume that

$$
w_{j}=e_{2 j-1}-i e_{2 j},
$$

with $1 \leq j \leq k$, and that $W=\sum C w_{j}$. Set $h_{j}=X_{2 j-1}-i e_{2 j}, 1 \leq j \leq k$. Then $\mathrm{t}=\sum R h_{j}, \quad$ is $\quad \mathrm{a}$ maximal Abelian sub-algebra of $\mathfrak{s o}(V)$. Let $\mu_{j} \in \mathfrak{t} *$, be defined by $\mu\left(h_{j}\right)=\delta_{j k}$. A direct calculation yields

$\mu(h) w_{1} \wedge \cdots \wedge w_{k}=\left(i\left(\mu_{1}+\mu_{2}+\cdots+\mu_{k}\right) / 2\right) w_{1} \wedge \cdots \wedge w_{k}$,

and

$$
\mu(h) \gamma\left(w \sigma_{j}\right)=-i \mu_{j}(h) \gamma\left(\sigma w_{j}\right) \mu(h),
$$

where $h \in \mathbf{t}$. This implies that

(*) the weights of the Lie algebra $t$, on $S(V)$, are precisely the linear functional

$$
\begin{gathered}
i\left(\mu_{1}+\mu_{2}+\cdots+\mu_{k}\right) / 2-\left(\mu_{i_{1}}+\mu_{i_{2}}+\cdots+\mu_{i_{p}}\right), \\
1 \leq i_{1}<\cdots<i_{p} \leq k,
\end{gathered}
$$

and each occurs with multiplicity 1.

Notice that this last is independent of our choice of $\gamma^{ \pm}$, when $n$, is odd. If $n$, then $\mathfrak{s o}(V)$, is semi-simple and is the Lie algebra of the compact Lie group

$$
O(V)=\{g \in G L(V) \mid(g v, g w)=(v, w), \forall v, w \in V\},
$$

which is $S O(n)$, in the spinor endomorphisms version en $V$. Weyl's theorem implies that $G$, the connected, simply connected Lie group with Lie algebra, $\mathfrak{s o}(V)$, is compact. Also exp $\mathfrak{t}=T$, is a maximal torus in $G$, and $\mu$, integrates to a representation of $G$. Then $(\star)$, implies that the character of $\mu$, is independent of our choice of $\gamma^{ \pm}$. So the assertion of the proposition 3. 2. 1., has been proved in the when $n$, is odd.

We therefore confine our attention to the case when $n$, is even. It is clear that the spaces $S^{ \pm}(V)$, are invariant. Set $\mathfrak{a}=\mathfrak{s} \mathfrak{o}(V)$. We assume that $\operatorname{dim} V>2$ (in the case that $\operatorname{dim} V=2$, the result that we are proving is an easy exercise). We set

$$
\begin{aligned}
\Phi^{+}= & \left\{i\left(\mu_{r}-\mu_{s}\right) \mid 1 \leq r \leq s \leq k\right\} \\
& \bigcup\left\{i\left(\mu_{r}+\mu_{s}\right) \mid 1 \leq r \leq s \leq k\right\},
\end{aligned}
$$


Then $\Phi^{+}$, is a system of positive roots for $\Phi\left(\mathfrak{x}_{C}, t_{c}\right)$. The only dominant weight in $(\star)$ are

$-i\left(\mu_{1}+\mu_{2}+\cdots+\mu_{k}\right) / 2$, and

$-i\left(\mu_{1}+\mu_{2}+\cdots+\mu_{k-1}-\mu_{k}\right) / 2$. Then assertion for $n$, even now follows from the theorem of the highest weight.

Proposition 3.2.2 (F. Bulnes). The set of hypersurfaces $S$, conforms under holomorphicity and polarization of the fields inside each hypersurfaces our spin space $\mathrm{s}_{\text {NANO }}$ (see Figure 4(c)).

Proof. The corresponding holomorphic Chern-Simons theory establish the action

$$
\mathfrak{J}_{h C S}=\frac{1}{2} \int_{M} \Omega^{3,0} \wedge \operatorname{tr}\left(X^{0,1} \wedge \bar{\partial} X^{0,1}+\frac{2}{3} X \wedge X \wedge X\right),
$$

originated from the field

$$
F^{0,2}=\partial X^{0,1}+X^{0,1} \wedge X^{0,1}
$$

which need of Calabi-Yau manifolds. Being a Calabi-Yau manifold a spin manifold, as is mentioned above, then the related strings with the fields and their polarization (orbits of the space-time (see Figure 4(a)) need of $2 n$-dimensional manifolds embeddings into space-time. In every one of these manifolds subjacent Lagrangian submanifolds $\mathbb{L}$, that is to say, these shape special embeddings in a $C Y$ - manifold [10,28,33]. These are the required holomorphic hypersurfaces in a $C Y$ - manifold. As in this decomposition of $2 n$-dimensional submanifolds to appear inevitably $D$-branes (see Figure 4(b)), in these, subjacent Hilbert spaces like spaces of bundle sections $\wedge \mathrm{T}^{(0,1) *} \mathcal{S} \otimes \mathbb{E}^{a *} \otimes \mathbb{E}^{b}$, where $\mathbb{E}^{a}$, and $\mathbb{E}^{b}$, are holomorphic bundle of lines of corresponding gauge fields [34]. The tensor products $\mathbb{E}^{a^{*}} \otimes \mathbb{E}^{b}$, have as square elements of their endomorphic elements de $\mathrm{s}_{\text {NANO }}$.

Here the space of bundle sections $\wedge \mathrm{T}^{(0,1) *} S \otimes \mathbb{E}^{a *} \otimes \mathbb{E}^{b}$, is one of the mentioned in the image of the algebra $t *$, to the two components in the strings context of the field.

So that the intention becomes effective (that is to say, their Lagrangian of effective energy are applicable [20]), there must be a co-action between the conscience of the field that applies the intention and the conscience of the field of the object that is wanted to transform. Likewise this coercion must be realized in synchrony and tuning, with the same intensity for both fields. This way, the proposition 3.2.2, establishes that the nano-space is the zone where initiates an organized transformation of nano type.

\section{Results}

In mathematics, an isotropic manifold is a manifold in which the geometry doesn't depend on directions. A simple example is the surface of a sphere. This directional independence grants us freedom to generate a quantum dimension process, since it does not import what direction falls ill through a string, the space is the same way affected and is presented the same aspect in any direction that is observed creating this way their isotropy.

The importance of this isotropy property in our spin manifold, helps us to establish that the transformations applied to the space that are directed to use (awakening) their nano-structure are did through an organized transformation that introduces the time as isotropic variable, creating a momentary timelessness in the space where the above mentioned transformation is created. Then the intentionality like an organized transformation is a co-action compose by field that act to realise the transformation of space and the field of the proper space that is transformed. Then the symplectic structure subjacent in $M$, haves sense.

Likewise, if $\mathcal{T}$, is a transformation on the space $M$, whose subjacent group $G$, have endomorphisms $\sigma_{1}, \cdots, \sigma_{n}$, such that $\sigma_{1} \mathcal{T}(\mathbb{M}), \cdots, \sigma_{n} \mathcal{T}(\mathbb{M})$, are isotropic then the infinite tensor product of isotropic submanifolds is a isotropic manifold, and is a organized transformation equivalent to tensor product of spin representations $\sigma_{1} \mathcal{T}(M) \otimes \cdots \otimes \sigma_{n} \mathcal{T}(M) \otimes \cdots, \quad[6,14]$.

Proposition (Bulnes) 4.1. The length of the quantum path that represents the angular relation between two "connected" spin manifolds (spins) is of the inferior or-

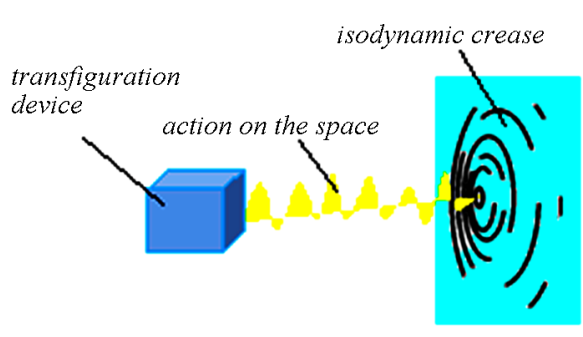

(a)

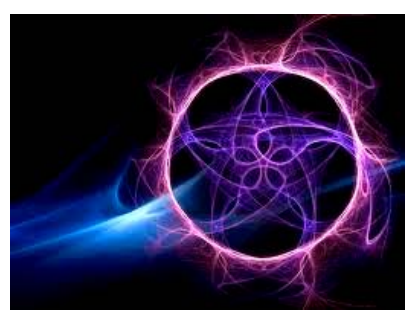

(b)

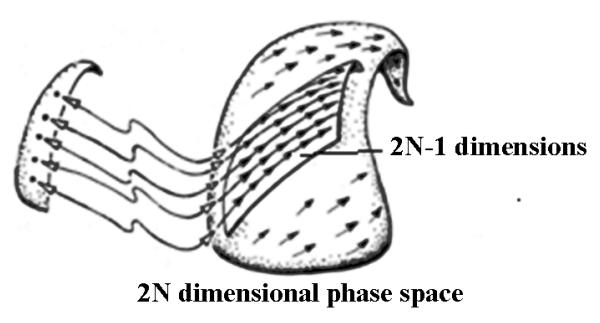

(c)

Figure 4. (a) Waking up to the space with a guided action. (b) Elementary quantum structure being content for the arrival of strings that are added. (c) $(2 N-1)$ - dimensional spin space and the $2 N$-dimensional phase space. 
der to $12^{-13} \mathrm{~cm}$.

Proof. Consider hypersurface $S$ (defined from (53) to (57)), defined in $\mathrm{M}^{2 n}$, such that $S \subset \mathcal{H}$, where $\mathcal{H}$, is the phase space defined in the structure (38). One element of this hypersurface comes given by $\Theta^{(n-k)}=\in_{a_{1} \cdots a_{N-k}} \mathrm{~d} W^{a_{1}} \wedge \cdots \wedge \mathrm{d} W^{a_{N-K}}$, but the global holomorphic form on the ambient space is $\Omega^{(N)}=\epsilon^{i_{1} \cdots i_{N}} \mathrm{~d} Z_{i_{1}} \wedge \cdots \wedge \mathrm{d} Z_{i_{N}}$, which represent the infinitesimal volume of the microscopic space-time, if we consider the atomic scale. But in this scale the before volume have the value $10^{-27} \mathrm{~m}^{3}$. But this number is represented approximately by the AMU (Atomic Mass Unit), that is to say $1.6604 \times 10^{-27} \mathrm{~kg}$, [10] which is induced from the intention given by the action $\mathfrak{I}_{O_{c}}$, (Figure 4(b)) until the curve that measures the linking energy for nucleon in function of the mass number (Figure 4(b)). Then the infinitesimal volume element $\Omega^{(N)}$, to every particle that have received this intention is approximately the Max Planck length except in $10^{-3} \mathrm{~nm}$, that is a little volume of the pure intention transmitted in nanoseconds. Also the same argument to the hypersurface in the space $\mathcal{H}$ (considering the little surface $10^{-2} \mathrm{~nm}$ ) that is to say, the length $10^{-20} \mathrm{~m}$. Then by the (62) considering the invariance hypothesis to the considered measures we have

$\Omega^{(k)}=\frac{l_{\underline{\underline{Z}}_{1}^{a}} \cdots l_{\underline{\underline{Z}}_{\theta}^{\alpha}} \Omega^{(N)}}{l_{\underline{\underline{Z}}_{1}^{a}} \cdots l_{\underline{\underline{z}}_{\theta}^{\alpha}} \Theta^{(N-k)}}=\frac{\left(10^{-3} \mathrm{~cm}^{3}\right) 10^{-27} \mathrm{~cm}}{\left(10^{-2} \mathrm{~cm}^{2}\right) 10^{-18} \mathrm{~cm}}=10^{-13} \mathrm{~cm}$,

Then the path that connect the two spin manifolds have the length

$$
\int_{\Omega(\Gamma)} \Omega^{(k)}=\left(10^{-13} \mathrm{~m}\right) \times 9.3463,
$$

But $12^{-13} \mathrm{~cm}=9.3463 \times 10^{-15} \mathrm{~cm}=9.3463 \times 10^{-13} \mathrm{~m}=$ 0.0009346 .

Theorem 4.1 (Bulnes). The BRST-cohomology $H_{\infty}\left(\mathrm{G}_{1}, \mathrm{G}_{0}, \mathrm{M}\right)=0 \quad \forall m \neq 0$, and $m<l$, with $l=\operatorname{dim} \mathbb{M}$.

Proof. We apply the BRST-cohomology to the space-time M, with the following decomposition

$$
M=\left(\bigoplus_{\lambda} G_{\lambda}\right) \oplus G_{I_{0}},
$$

where $\mathrm{G}_{0}$, is the Fock space at zero center-of-mass momentum and $\mathrm{G}_{\lambda}$, are Verma modules whose highest weight vectors are obtained by repeated application of the creation operators in the full spectrum generating algebra.

According to the decomposition of $\mathbb{M}$, the semi-infinite cohomology also breaks up as

$$
\bigoplus_{\lambda} H_{\infty}\left(\mathcal{N} S, \mathbb{G}_{\lambda}\right) \oplus H_{\infty}\left(\mathcal{N} S, \mathbb{G}_{0}\right) \text {. We ignore } \mathbb{G}_{0},
$$

because it represents the permanent quantum energy that does possible all transformations from particles to parti- cles (energy support). Here space $\mathcal{N}$, is the virtual zone of the space-time (Higgs fields). From now on and we focus on $H_{\infty}\left(\mathcal{N S}, \mathbb{G}_{1}\right)$, where $G_{\text {, }}$, is a fixed $\mathbb{G}_{\lambda}$. Consider the relative subcomplex consisting of vectors $\omega \in H \quad$ Fock space satisfying $b_{0} \omega=O_{c}^{0} \omega=0$. The induced differential in this subcomplex is easily seen to be the operator $Q$, (extended BRST-operator) who include the more and deep action of $O_{c}$, reflected in (32). The cohomology of this complex is nothing but the relative semi-infinite cohomology $H_{\infty}\left(\mathcal{N} S, \mathcal{N} S_{0}, G\right)$, where $\mathcal{N} S_{0}$, is the subalgebra generated by $O_{c}^{0}$. Then there exists a filtration of this complex giving rise to a spectral sequence converging to

$H_{\infty}\left(\mathcal{N} S, \mathcal{N} S_{0}, G_{r}\right)$, whose $\mathrm{E}_{1}$, term obeys $\mathrm{E}_{1}^{m}=0$, for $\quad m<0$. Therefore $H_{\infty}^{m}\left(\mathcal{N S}, \mathcal{N} S_{0}, \mathbb{G}_{\mathrm{G}}\right)=0, \quad$ for $m<0$. What happen with the case $m>0$ ? We consider the "Poincare duality" theorem [10], which says

$$
H_{\infty}^{m}\left(\mathcal{N} S ; \mathcal{N} S_{0}, G_{r}\right)=H_{\infty}^{-m}\left(\mathcal{N} S ; \mathcal{N} S_{0}, G\right)
$$

allows us to conclude that $H_{\infty}^{m \neq 0}\left(\mathcal{N} S, \mathcal{N} S_{0}, G\right)=0$, $\forall m<l$.

Lemma 4. 1 (Bulnes). Let $\mathrm{M}=M \times I_{t}$, the unlimited space of the quantum space (Fock space [24]). A particle $x(t)$, that is focalized by a bad evolution given for the energy load function $w(t, s)$, comes given for

$$
x(s)=\int_{\mathcal{M}} \phi\left(x^{\prime}\right) x(t) \mathrm{d} x(t)=\int_{-\infty}^{+\infty} \delta(t-s) x(t) \mathrm{d} t,
$$

Then to time $t=s$, begin the singularity.

Proof. [11,12].

If we consider a conscience operator with singularity $O_{c}(*)$, for the presence of an energy load $w(s, t)$. Then the elimination of the singularity $*(s)$, comes given by the theorem [12]:

$$
\begin{aligned}
x(t) & =\text { correction }+ \text { restoring }=\int_{X(C)} O_{c}(*(s)) w(t, s) \mathrm{d} t \\
& =\operatorname{dim} \Lambda(\alpha) \int_{C}\left\{\frac{1}{A} \prod_{j=1}^{\infty}\left\{\int_{-\infty}^{+\infty} \phi\left(n_{j}\right) F\left(n_{j}\right)\right\} \mathrm{d} x(t)\right\},
\end{aligned}
$$

where $\operatorname{dim} \Lambda(\alpha)$, is the Neumann dimension corresponding to the Weyl camera of the roots $\alpha_{j},[12,23]$ used in the rotation process to eliminate the deviation [8], created by the singularity.

Proof. [11,12].

Arguments for algebras and homotopies, and their meetings we can demonstrate the nature of sum "correction + restoring" established in (75) that haves their realization by the path integral established in (10) y (11). Then we can enunciate the pair of quantum integral transforms as [12]

$$
\mathcal{Q}\{x(t)\}=\int_{X(C)} O_{c}(x(t)) w(t, s) \mathrm{d} s=\int_{X(C)} O_{c}(x(t)) \mathrm{d} \phi,
$$




$$
\begin{aligned}
\mathcal{Q}^{-1}\{*(t)\} & =\operatorname{dim} \Lambda(\alpha) \int_{X(C)} O_{c}(*(s)) w(t, s) \mathrm{d} t \\
& =\int_{X(C)} O_{c}(*(s)) \mathrm{d} x,
\end{aligned}
$$

Let $\Phi$, be the potential that the integral transform (76) have to realize (that is to say, there is a realization $\mathrm{R}$ (goal or target)) a quantum transformation of a region of the space-time $C$, from the field $X$. The integrals of line that derived through a bundle of lines $O(1,1)$, are projectivized in $C$, since there is a topological space "sky" $Q_{x}$, such that $Q_{x} \cong \mathbb{P}^{1} \times \mathbb{P}^{1}$. Likewise one integral of line (of the type described in (44)), is the realization $R=\oint \alpha(\xi(\beta(x(s))))$. Then the exact succession (46) is translated for the potential of this quantum realization in the commutative diagram $[6,11]$ :

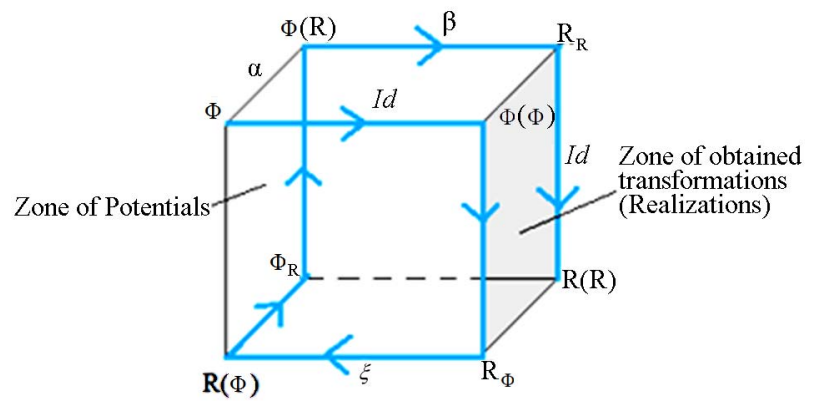

where $\alpha, \beta$, and $\xi$, are differentiable maps in all the space $C$.

Consider to $\Phi$, a set of transformation potentials of the space $M$, such that

$$
\begin{gathered}
\Phi_{\xi}=\left\{\varphi \in C^{\infty}(\Omega(\Gamma), \mathbb{R}) \mid \varphi \circ \xi=\int_{\Omega(\Gamma)} D_{\varphi} \xi,\right. \\
\left.\forall D_{\varphi}=\Delta_{F} \varphi, \text { and } \xi \in \mathrm{TM} *\right\},
\end{gathered}
$$

Proposition (Bulnes) 4.2. Let $\Phi_{\xi}$, be the set of potentials in nano. $\Phi_{\xi}$, is potentiality if only if $\xi$, is a fiber through which $\varphi$, is a potential.

Proof. Let $\mathrm{s}_{N A N O}$, the nano of the space-time $\mathbb{M}$. Inside of nano, the force of potential is given by $\Delta_{F} \varphi=\delta\left(x-x^{\prime}\right) \varphi$. Then their conscience operator $O_{c}$ (by the property $i v$, given in the Section 2.1) is said force. But by the definition of our conscience operator $O_{c}$, is a fiber of the corresponding Lagrangian operator that involves states. In terms of the space TM*, this is a Hamiltonian which satisfies the $\operatorname{grad} \varphi$, in question. But $\varphi \circ \xi$, is the potential that have the zone $\Omega(\Gamma)$, in the space-time. In special, along of anyone trajectory $\Gamma$, this camera $\Phi_{\xi}$, is a potentiality. The reciprocate, if $\Phi_{\xi}$, is potentiality then $\xi$, is a fiber through which $\varphi$, is a potential, is deduced immediately applying the corresponding path integral whose potential is $\varphi$.
Then $\Phi$, is the sum over all basic states corresponding to a fixed choice of $\phi^{0},\left(\gamma^{\prime} s\right.$, and $\left.\phi^{\prime} s\right)$, of the corresponding "amplitude" (to be defined shortly):

$$
\Phi=\sum_{\text {all basic states } \phi \text { of } \Gamma} \Phi_{\phi},
$$

Note. The true amplitude of the process would involve a sum over all states, when the values of $\phi^{0}$, varies on the internal vertices while the state of the boundary $\phi$, is fixed. The sum over values on the edges amounts to a contraction process (traces etc.).

Now $\Phi_{\phi}, \quad\left(\Phi_{\phi}\right.$, in (86) or also $\Phi_{\alpha}$, in of the section IV) is a product over the contributions $\Phi_{\phi}(v)$, over the vertices of $\Gamma, n$, internal and $m$, boundary type.

For an internal vertex $v$, we have:

$$
\Phi_{\phi}(\mathrm{v})=\left(\prod_{e \in i n(\mathrm{v})} \phi(\mathrm{e})\right) \psi_{\mathrm{v}}, \psi_{\mathrm{v}}=\left\langle\gamma_{\mathrm{v}}, \underset{\mathrm{e} \in \text { out }(\mathrm{v})}{\otimes} \mathrm{d} x^{\mathrm{R}(\mathrm{e})}\right\rangle,
$$

where $\operatorname{in}(\mathrm{v})$ (or $\operatorname{out}(\mathrm{v}))$ denotes the set of incoming (outgoing) edges of the vertex $v$, and the shorthand notation $\phi=\phi^{(1)}$, was used since $\phi^{0}$, is fixed within this state-sum.

Towars an "propagation amplitude" interpretation (as have established in the space $\Phi_{\xi}$ ) replace the evaluation pairing with the inner product (,), such that the above basis $\left\{\partial_{i}\right\}_{i}, i=1, \cdots, n$, be orthonormal. Also collect the "in" and "out" products, introducing the following terminology.

Definition 4.1. For any basic state $\phi$, of the graph $\Gamma$ :

$$
\phi_{\text {in }}=\left(\prod_{\mathrm{e} \in \text { in }(\mathrm{v})} \phi(\mathrm{e})\right), \phi_{\text {out }}=\underset{\mathrm{e} \in \operatorname{out}(\mathrm{v})}{\otimes} \phi(\mathrm{e}),
$$

are called the in and out states of the scattering process at the vertex $v$.

Very process that gives beginning to the shape singularity are related with the scattering amplitude obtained from the internal vertex inside space, body or object that is wanted to realize a transformation by nano (see Figure 5). Of fact, some invasive methods in nanotechnology (like the use of nanobots, follows this idea) require that the transformation of the space $M$, initiate inside of the space, object of the transformation. Nevertheless, this idea is implicitly given with the intentionality and the operators $O_{c}$, since require of the two actions, the action from field that is applied and the action of the proper field of the space, object or body. Being this important we have:

Proposition 4.3.

$$
-\Phi_{\phi}(\mathrm{v})=\left(\phi_{\text {out }}(\mathrm{v}), \operatorname{ad}_{\phi(\mathrm{v})}\left(\psi_{\text {in }}(\mathrm{v})\right)\right)
$$




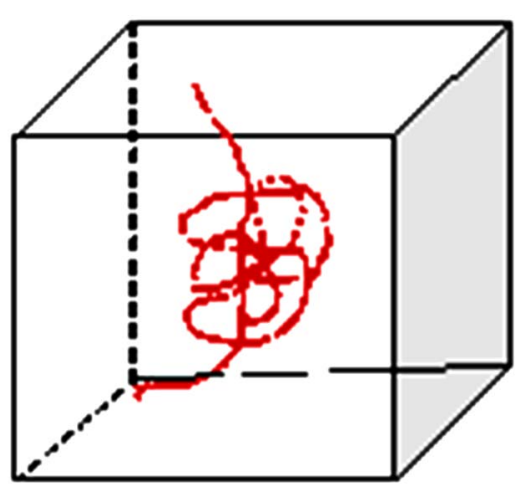

(a)

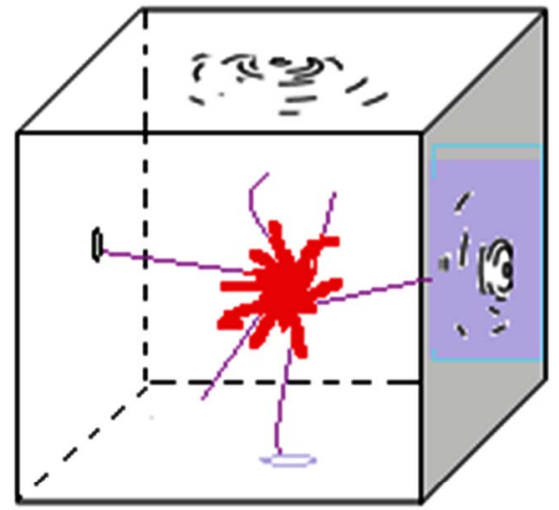

(b)

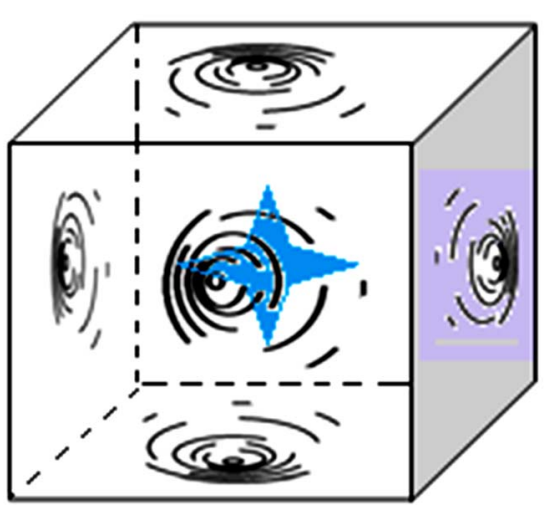

(c)

Figure 5. The intention is applied and begins an inner transformation of the space $M$ (the singularity is born). The action of the field (from observer) is transmitted to the field of the proper space that we want to transform. Using direct transform, the singularity is implanted in the space by the intention of the field (a)-(c). For the case of the inverse transform we annul the transmission of the conscience in the singularity $O_{c}(*)$, and focus to the space $M$, by means of the operator $O_{c}(x(t))($ to revise the interjections of the figures in the inverse order).

is the scattering amplitude

$$
\left(\phi_{\text {out }}(\mathrm{v})\left|, \operatorname{ad}_{\phi(\mathrm{v})}\right|\left(\phi_{\text {in }}(\mathrm{v})\right)_{\mid v}\right)
$$

of the elementary process at internal vertex $v$ :

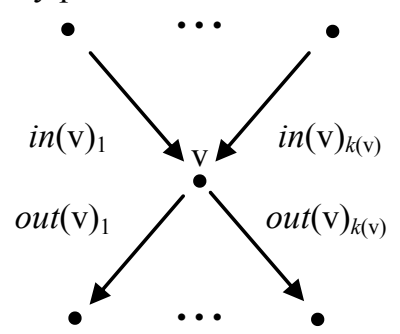

Proof. Here $\operatorname{ad} X(Y)=[X, Y]$, is the commutation bracket on differential operators. Therefore if $X$, and $Y$, commute, then $[X, \phi Y]=X(\phi) Y$. Now to retrieve the appropriate component, use the above inner product:

$$
\Phi_{\phi}(v)=\phi_{\text {in }}\left(\psi_{v}\right)=\left(\left[\psi_{\text {in }}(v), \gamma_{v}\right], \phi_{\text {out }}(v)\right),
$$

This can be put in the form of propagation amplitude establishing the above claim.

The integral transforms apply inverse methods of quantum re-compositions that produce quantum transformations "appropriate" to establish an answer of the states of energy of the part $M$, affected by singularity, and re-compose the space establishing an image of the field $X(\mathrm{MI})$, adapted in same resonance, quantum composition of the space points $X(t)$, of the whole space $M I$. This last for the synergic principle of the actions of the field (postulate 3, in [17]), and due to the field intention that has the proper matter of the space $M$, with tendency to an auto-ordering in the nano-level.

The answers between densities are realized in accordance with the correlation densities established in certain commutative diagrams that can be shaped by spaces $L^{2}$, on the space-time of the particles [35].

Coding this region of transition states of the corresponding Feynman diagram on a logic algebra $\mathcal{A}(\cap, \cup,-)$ (like full states or empty of electrons like particle/wave, is to say, $\phi(0)=0$ (is not the particle electron, but is like wave) $\phi(1)=1$ (is not the wave electron, but is like particle) and their complements), where the given actions in [8], are applied and re-interpreting the region of the space-time of the particles like a electronic complex of a hypothetical logic nano-floodgate (that is to say, like a space $L^{2}$, with a logic given by $\mathcal{A}(\cap, \cup,-)$, with values in $\mathcal{M}_{0,1}$ $[8,17]$, on their transition states), we can define the Feynman-Bulnes integrals, $[6,7,17,23]$, as those that establish the transition amplitude of our systems of particles through of a binary code that realizes the action of correction and restoration of the field established in [8]. Likewise a Feynman-Bulnes integral [17,36,37], is a path integral of digital spectra with the composition of the fast Fourier transform of densities of states of the corresponding Feynman diagrams. Thus, if $\phi_{1}, \phi_{2}, \phi_{3}$ and $\phi_{4}$, are four transitive states corresponding to a Feynman diagram top holes of the field $X(M)$, then the path integral of Feynman-Bulnes is [36]:

$$
\begin{aligned}
I & =\int_{\phi^{-}}^{\phi^{+}} \phi_{n_{1}} F\left(n_{1}\right) \phi_{n_{2}} F\left(n_{2}\right) \phi_{n_{3}} F\left(n_{3}\right) \phi_{n_{4}} F\left(n_{4}\right) \\
& =0001101001 \cdots,
\end{aligned}
$$

The integrals of Feynman-Bulnes, establish the amplitude of transition to that the input of a system with signal $x(t)$, can be moved through of a synergic action of electronic charges $\mathfrak{I}$, doing through of pre-determined waves functions by $L(x(t))$, and encoded in a binary 
algebra (pre-defined by states $\phi(0), \phi(0)$, and $\phi(1))$, (in the kernel of the space of solutions of the wave equation $\square_{x} \Delta_{F}^{A A^{\prime}}=\nabla^{A A^{\prime}} \delta\left(x-x^{\prime}\right)$ [16]) of a point to other into of a circuit $\Gamma_{j}$. Their integral it is extend to all space of paths or re-walked included into of the region of Lagrangian action $\Gamma\left(=\bigcup_{j}^{k}\left(\bigcap_{j}^{k} \Gamma_{j}\right)\right)$ with a topology of signals in $L^{2}(\cap, \cup,-)[4,6]$. If we want corrective actions for stretch $\Gamma_{j}$, of a path $\Gamma$, these we can realize them using diagrams of strings of corrective action using the direct codification of path integrals with states of emission-reception of electrons (by means of one symbolic cohomology of strings [6,25]). Then the evaluation of the Feynman-Bulnes integrals it reduces to the evaluation of the integrals: $I(\Gamma, \Omega)=\int_{C_{\Gamma}^{0}(\Psi)} \omega(\Gamma)$, where $\Omega$,

is the orientation of $C_{\Gamma}^{0}(\Psi), \Psi$, is the corresponding model of graph used to correct after of identify the singularity of the field $X$, that distorts it. For example, observe that is can to null the corrective action of erroneous encoding through of a sub-graph:

$$
\begin{aligned}
\int_{\Gamma_{0}} \omega(\Gamma) & =1 \cup-1 \cup 0 \cup[\phi(0) \cup 0-\phi(1)] \cup 1 \\
& =0-\phi(1) \cup 1=-1 \cup 1=1+(-1)=0,
\end{aligned}
$$

The corresponding equation in the cohomology of strings is $[6,38]$ :

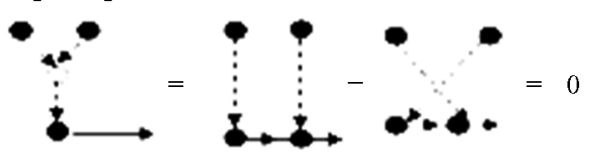

The quantum transformations due this inverse action in this level of deep stay established by the logic defined in $\mathcal{M}_{0,1}$ (acquaintance as cyberquantum algebra for their applications in cybernetic), based in path integrals belonging to the cohomological space

$H^{1}\left(\mathbb{P}^{\top} M^{ \pm}, O(-k)\right)$, and whose action organize and correlating all movements of particles $x(s)$, in an symphony that devolve of initial shape of the matter.

Proposition (Bulnes) 4.4. Using organized transformations as given in $\sigma_{1} \mathcal{T}(\mathbb{M}) \otimes \cdots \otimes \sigma_{n} \mathcal{T}(\mathbb{M}) \otimes \cdots$, we can to establish that the state of all particles in set, is their corresponding Fock image [39].

Proof. Is necessary to apply the group of permutations $\sigma_{j}$, on at least $n$, components of the space $\mathrm{H}=\mathrm{H}_{1} \otimes \cdots \otimes \mathrm{H}_{n}$, with $\mathrm{H}_{1}=\cdots=\mathrm{H}_{n}$.

Inside of the Fock space begins a realization of the potential of the intention, since the Fock pure state involves all the states of particles of the space, object of the transformation.

We consider $E_{F I S M A T}$, the space of the all physical applications to make nanotechnology, (set of acknowledges in mathematics and physics: "Remember that the intention must know their goal") [6]. We will define as homomorphism of certain class to the application on the space of knowledge applications $E_{\text {FISMAT }}$, and with values in $\Phi_{\alpha}\left(E_{\text {FISMAT }}\right)$, which we will identify as the set of advance useful in the nanotechnology creation in the class $\alpha$ [40,41].

If we consider an action like the product of diverse actions $\mathfrak{I}_{1} \times \cdots \times \mathfrak{I}_{n}$, determined in a technology $t_{\sigma}$, the integral operator of corresponding execution will come determined for

$$
\begin{aligned}
& \boldsymbol{e x p}_{1}\left(\boldsymbol{e x p}_{2} \cdots\left(\boldsymbol{e x p}_{n}\right) \cdots\right): \mathfrak{I}(x(s)) \\
& =\int_{E_{n}}\left(\cdots\left(\int_{E_{1}} \mathfrak{I}(x(s)) \mu_{1}\right) \cdots \mu_{n}\right) \\
& =\int_{E_{1} \times \cdots \times E_{n}} \mathfrak{I}(x(s)) \mu_{1} \cdots \mu_{n},
\end{aligned}
$$

In this case the metric evaluation of the nanotechnology is carried out in an $n^{n}$-dimensional space. If in particular $E=E_{F I S M A T}$, then $\mathfrak{I}=\phi$, with $\phi$, a homeomorphism (neologism of technologies), then

$$
\operatorname{exe}\left(t_{\delta}\right)=\operatorname{exe}\left(\phi_{\gamma} \Phi_{\alpha}(E)\right)=\int \phi_{\gamma} \Phi_{\alpha} \mathfrak{I}\left(\Phi_{\alpha}\left(E_{F I S M A T}\right)\right) \mu_{\alpha},
$$

$\forall x(s) \in \Phi_{\alpha}\left(E_{F I S M A T}\right)$, and $\mu_{\alpha}$, the corresponding measure on the technological constituted wealth this, for the corresponding curves of technological profiles according to the evolution of technology $[6,40,41]$.

Proposition (Bulnes) 4.5. The image of homomorphism (88) is a transmission of intention from $\alpha$, to $\gamma$, with connected $\alpha$.

Proof. By the proposition 4.2, with the properties of operator $O_{c}$ (Section 2.1), and proposition 4.3, the result is risked.

We have considered to the technologies like holistic entities of devices, components and implements of any physical nature that under laws of the quantum mechanics and field theory contained in $E_{\text {FISMAT }}$, they act under an operator for the design of experiments it has more than enough minimum trajectories of evolution of the process of these experiments and their evaluation of these technologies along such trajectories by means of functional corresponding.

Basing us on this thesis, it is need to characterize a space topologic of rehearsals or tests and re-tests of technologies whose evaluation should be carried out through instrumentation that throws measures and values in topological spaces $L^{2}$ [35]. These measures should spread to a limit of nano-technological application (bench mark [12]) which is a measure inside the space of functions $\mathfrak{L}^{2}(G)$ [40,42].

The relation between the space $\mathrm{S}_{N A N O}$, and the Banach space $E_{F I S M A T}$, is endow of Hilbert structure to the space $E_{\text {FISMAT }}$, doing that their endomorphism let measurable in the space $L^{2}(G)$. The instruments are in 
the space of measures $\mathfrak{L}^{2}(G)$.

Let us consider to $X_{L A B}$, like the space of rehearsals or tests of technological process. Let us endow $X_{L A B}$, of a structure of Hilbert space also of the Hamiltonian structure that have due at their ergodicity (see Ergodic theory of dynamical systems (principle 3), [39,43]), then the space of true propositions verified in $X_{L A B}$, satisfies

$$
\Phi_{\alpha}\left(X_{L A B}\right) \text { Implies tautologically } \tau_{a}(X(f)),
$$

$\forall f \in \mathfrak{L}^{2}(G), \quad \tau_{\alpha} \in \Lambda_{\text {SYSTEM }}\left(\Phi_{\alpha}, t_{\sigma}\right), \quad$ (the transfer technologies operator), and where belonging to a class (that is to say, applicable to a certain class of technologies) they are isometries from $E_{\text {FISMAT }}$, to $X_{L A B}$, having that for a variety of technological applications and with the relevancy of considering widespread measures of the technological applications, we can consider

$$
\begin{aligned}
& \left\langle\tau_{\alpha} X(f), t_{\gamma}\right\rangle \\
& =\int_{G} \Im\left(t_{\delta} \circ t_{\sigma} \circ t_{\delta}^{-1}\right)\left(\phi_{\sigma}\left(t_{\eta}\right) \phi_{\sigma}\left(t_{\gamma}\right) \phi_{\sigma}^{-1}\left(t_{\eta}\right)\right) \mu_{\sigma},
\end{aligned}
$$

which bears according to the topology of $G$ (as topological group), the approach of following inequality:

$$
\left\|\tau_{\alpha} X(f) t_{\gamma}\right\| \leq\left\|\log \phi_{\sigma}\left(t_{\eta}\right)\right\|^{a}\left\|\log \Im\left(t_{\sigma}\right)\right\|^{b},
$$

with values in $a=b=2$. The controls to $\tau_{\alpha} X(f) t_{\gamma}$, are given for $\log \phi_{\sigma}\left(t_{\eta}\right)$, and $\log \Im\left(t_{\sigma}\right)$, [35,40].

But the one that are isometries in the context of $\mathfrak{Q}^{2}(G)$, it bears to that in the space of technological applications under the technology transfers are satisfied:

Theorem (Bulnes) 4.3. 1) $E_{F I S M A T}$, is isometric to $X_{L A B}$,

2) $\tau_{\alpha}\left(E_{F I S M A T}\right)=\Phi_{\alpha}\left(X_{L A B}\right)$.

Proof. Consider the space

$$
\tau_{\alpha}\left(E_{F I S M A T}\right) \cap \Phi_{\alpha}\left(X_{L A B}\right)=\{0\},
$$

That which is equal in the sense of the transfer of technologies to that $\tau_{\alpha} \in \Lambda_{\text {SYSTEM }}$,

$$
\left\langle\tau_{\alpha}\left(t_{\sigma}\right), t_{\gamma}\right\rangle-\left\langle t_{\sigma}, \tau_{\alpha}\left(t_{\gamma}\right)\right\rangle=0,
$$

$\forall t_{\sigma}, t_{\gamma} \in \mathbb{G}$ [4]. Likewise, in the context of the spaces $\operatorname{Lin}(\mathrm{H}, \mathrm{H})$, the relationship of previous symmetry that in the space $\Lambda_{\text {SYSTEM }}$, is transcribed in the environment of the integral operators belonging to $\mathfrak{L}(\mathrm{H})$, on the infinite straight line $\mathbb{R}$, as

$$
\int_{\mathfrak{R}} \Phi_{\alpha}(x(s)) f(s)-F(s) \phi_{\alpha}\left(t_{\sigma}(x(s))\right) \mathrm{d} s=0,
$$

This integral is the integral of execution of the actions of each component o particle given in generalized coordinates $\left(q^{1}, \cdots, q^{n}\right)$, that they specify the configuration of us dynamical system in certain $n$-dimensional space [4]. We consider Hamilton's principle of the minimal action on these generalized coordinates then

$$
\int_{t_{1}}^{t_{2}}\left(\delta T+Q_{i} \delta q^{i}\right) \mathrm{d} t=0
$$

By Ergodic theory is not difficult prove that the measure on any curve in $M=\mathbb{R}^{6 N}$ ( $N$, is the number of particles) with the measure $\mathrm{d}^{3 N} q \mathrm{~d}^{3 N} p$ (well-known to be invariant under the Hamiltonian flow (Lioville's theorem)), is a measure in $\mathfrak{L}^{2}(G)$ [40,42]. This measure has a restriction to $\Omega_{E}$ (domain of energy of $M$ ) given formally by

$$
\mu_{E}(F)=\int_{F} \delta(H(p, q)-E) \mathrm{d}^{3 N} p \mathrm{~d}^{3 N} q,
$$

where $F$, is a measurable set of $\Omega_{E}$, and $H(p, q)$, is the Hamiltonian.

But (94) for other side, is the Parseval's theorem for the isometries group in $\mathfrak{L}(\mathrm{H})$. This induces us a symmetrical structure in the space $\Phi_{\alpha}\left(E_{F I S M A T}\right)$, that extended to the infinitude of the space $E_{\text {FISMAT }}$, is obtained

$$
\begin{aligned}
& \Lambda_{\text {SYSTEM }}\left(\Phi_{\alpha}\left(E_{\text {FISMAT }}\right), t_{\sigma}\right) \\
& =\operatorname{Lin}(H, H) \oplus \operatorname{Lin}(H, C)=B(H, H)
\end{aligned}
$$

where $B(\mathrm{H}, \mathrm{H})$, is the space of enclosed operators, with bench marks $N \leq\left\|\log \phi_{\gamma}\left(t_{\eta}\right)\right\|^{2}$, which are integrables mayoralties in the ways or Hermitian forms $\left\langle\tau_{\alpha}\left(t_{\sigma}\right), t_{\gamma}\right\rangle$, then $X_{L A B}$, is isometric to $E_{\text {FISMAT }}$, and it follows (a), sentence. For other side, $\Lambda_{\text {SYSYEM }}\left(\Phi_{\alpha}\left(E_{\text {FISMAT }}\right), t_{\sigma}\right)$, is the integrals space with coefficients in $\Phi_{\alpha}\left(X_{L A B}\right)$, and isomorphic to space $\tau_{\alpha}\left(t_{\sigma}(E)\right)$, the which is isomorphic to space

$\Phi_{\alpha}\left(E_{\text {FISMAT }}\right)$, when the integral is evaluated in points of the Banach space. Therefore

$$
\int_{G}\left[\Phi_{\alpha}\left(X_{L A B}\right)-\tau_{\alpha}\left(E_{F I S M A T}\right)\right] \mu_{\sigma}\left(\Im\left(t_{\sigma}\right)\right)=0,
$$

This integral is true for all technology application $t_{\sigma} \in \mathbb{G}$, for dual spaces in spectrum of technologies. Therefore it follows (2).

\section{Conclusions}

The future of the nanotechnology is the creation of the organized transformations on space-time, matter and energy using codes that are born of quantum intention of the field that we want to apply to. In the foreseen exposition along of the Sections 1, 2 and 3, it is demonstrated that nanotechnology processes are the same with that intention processes where in this case we call intentionality. This property is formulated with integration of two elements the field of which realizes the transformation 
and the object which is applied in this transformation. This defines the organized transformation in nanotechnology and the actions involved which shape the intention.

But also this intention realizes an integration of the field of the proper object to transform with the field that realizes this transformation. Cause and effect shape only one unit in this process. In the nanotechnology theory, it is necessary to realize that one third unification of the time and space considering a shortest of uncertainly in all the quantum process to define the synchronicity must be born in more studies and mathematical research of the motivic cohomology, K-theory, Lie infinite theory and motives theory applied to the QFT. In the nanotechnology process it will be fundamentally legitimized through the quantum electrodynamics and their new version that is necessary to obtain this synchronicity, given the principles to the advanced process that requires the teleportation, quantum communication and other phenomena that will be the next technologies to interstellar step and survivor of the humanity. In this work the maximum principle is included that must govern all the process in nanotechnology which is the intentionality.

\section{Acknowledgements}

I am grateful for the financing support offered by $D e$ metrio Moreno Árcega, M. in L. and Principal of the Technological Institute of High Studies of Chalco (TESCHA).

\section{REFERENCES}

[1] J. E. Marsden and R. Abraham, "Manifolds, Tensor Analysis and Applications," Addison-Wesley, Massachusetts, 1993.

[2] C. Chevalley, "Theory of Lie Groups," Princeton University Press, Princeton, 1946.

[3] F. E. Burstall and J. H. Rawnsley, "Twistor Theory for Riemannian Symmetric Spaces," Springer Verlag, New York, 1990. http://dx.doi.org/10.1007/BFb0095561

[4] F. Bulnes, "Analysis of Prospective and Development of Effective Technologies through Integral Synergic Operators of the Mechanics," The $14^{\text {th }}$ Scientific Convention of Engineering and Arquitecture: Proceedings of the 5th Cuban Congress of Mechanical Engineering, 2-5 December 2008, Havana.

[5] W. Rudin, "Functional Analysis," McGraw Hill Education, New York, 1973.

[6] F. Bulnes, H. F. Bulnes, E. Hernandez and J. Maya, "Diagnosis and Spectral Encoding in Integral Medicine through Electronic Devices Designed and Developed by Path Integrals," Journal of Nanotechnology in Engineering and Medicine, Vol. 2, No. 2, 2011, Article ID: 021009.

[7] F. Bulnes, "Theoretical Concepts of Quantum Mechanics," In: M. R. Pahlavani, Ed., Correction, Alignment, Restoration and Re-Composition of Quantum Mechanical
Fields of Particles by Path Integrals and Their Applications, InTech, Rijeka, 2012.

http://www.intechopen.com/books/theoretical-concepts-of -quantum-mechanics/correction-alignment-restoration-an d-re-composition-of-fields-of-particles-by-path-integralsand-the

[8] M. Alonso and E. Finn, "Fundamental University Physics, Volume III: Quantum Statistical Physics," Addison-Wesley Publishing Co., Massachusetts, 1968.

[9] R. P. Feynman, "Space-Time Approach to Non-Relativistic Quantum Mechanics," Reviews of Modern Physics, Vol. 20, No. 2, 1948, pp. 367-387. http://dx.doi.org/10.1103/RevModPhys.20.367

[10] F. Bulnes, "Cohomology of Moduli Spaces in Differential Operators Classification to the Field Theory (II)," Proceedings of FSDONA-11 (Function Spaces, Differential Operators and Non-linear Analysis, 2011), Vol. 1, No. 12, 2011, pp. 1-22.

[11] F. Bulnes, H. F. Bulnes and D. Cote, "Symptom Quantum Theory: Loops and Nodes in Psychology and Nanometric Actions by Quantum Medicine on the Mind Mechanisms Programming Path Integrals," Journal of Smart Nanosystems in Engineering and Medicine, Vol. 1, No. 1, 2012, pp. $97-121$.

[12] F. Bulnes, "Quantum Intentionality and Determination of Realities in the Space-Time through Path Integrals and Their Integral Transforms," In: P. Bracken, Ed., Advances in Quantum Mechanics, InTech, Rijeka, 2013.

http://www.intechopen.com/books/advances-in-quantummechics/quantum-intentionality-and-determination-of-rea lities-in-the-space-time-through-path-integrals-and-t

[13] H. B. Lawson and M. L. Michelsohn, "Spin Geometry," Princeton University Press, Princeton, 1989.

[14] J. Schwinger, "Particles, Sources, and Fields," Vol. 1, 3rd Edition, Advanced Book Program, Perseus Books, Massachusetts, 1998.

[15] R. P. Feynman, R. B. Leighton and M. Sands, "Electromagnetism and matter," Vol. II, Addison-Wesley, Massachusetts, 1964.

[16] F. Bulnes and M. Shapiro, "On General Theory of Integral Operators to Analysis and Geometry (Monograph in Mathematics)," 2007.

[17] F. Bulnes, H. F. Bulnes, E. Hernandez and J. Maya, "Integral Medicine: New Methods of Organ-Regeneration by Cellular Encoding through Path Integrals applied to the Quantum Medicine," Journal of Nanotechnology in Engineering and Medicine, Vol. 1, No. 7, 2010, Article ID: 030019 .

[18] D. McDuff and D. Salamon, "Introduction to Symplectic Topology," Oxford University Press, Oxford, 1998.

[19] I. S. Sokolnikoff, "Tensor Analysis: Theory and Applications," Wiley and Sons, New York, 1951.

[20] B. R. Holstein, "Topics in Advanced Quantum Mechanics," Addison-Wesley Publishing Company, Cambridge 1992.

[21] A. Kapustin, M. Kreuser and K. G. Schlesinger, "Homological Mirror Symmetry: New Developments and Perspectives," Springer, Berlin, 2009. 
[22] Y. Aharonov and D. Bohm, "Significance of Electromagnetic Potentials in Quantum Theory," Physical Review, Vol. 115, No. 3, 1959, pp. 485-491. http://dx.doi.org/10.1103/PhysRev.115.485

[23] F. Bulnes, "Doctoral Course of Mathematical Electrodynamics," In: National Polytechnique Institute, Ed., Appliedmath 3: Advanced Courses, Proceedings of the Applied Mathematics International Congress, 25-29 October 2006, México.

[24] E. R. LeBrun, "Twistors, Ambitwistors and Conformal Gravity," In: T. N. Bailey and R. J. Baston, Eds., Twistors in Mathematics and Physics, Cambridge University, Cambridge, 1990, pp. 71-86.

[25] L. P. Hughston and W. T. Shaw, "Classical Strings in Ten Dimensions," Proceedings of the Royal Society of London Series A: Mathematical and Physical Sciences, Vol. 414. No. 1847, 1987, pp. 423-431.

[26] F. Bulnes, "Penrose Transform on D-Modules, Moduli Spaces and Field Theory," Advances in Pure Mathematics, Vol. 2, No. 6, 2012, pp. 379-390. http://dx.doi.org/10.4236/apm.2012.26057

[27] R. Sobreiro, “Quantum Gravity,” InTech, Rijeka, 2012. http://www.intechopen.com/books/quantum-gravity

[28] P. Griffiths and J. Harris, "Principles of Algebraic Geometry," Wiley-Interscience, New York, 1994. http://dx.doi.org/10.1002/9781118032527

[29] M. Gross, D. Huybrechts and D. Joyce, "Calabi-Yau Manifolds and Related Geometries," Springer, Norway, 2001.

[30] J. Hoogeveen and K. Skenderis, "Decoupling of Unphysical States in the Minimal Pure Spinor Formalism I," 2010.

[31] N. Berkovits, J. Hoogeveen and K. Skenderis, "Decoupling of Unphysical States in the Minimal Pure Spinor Formalism II," 2009.

[32] T. W. B. Kibbe, "Geometrization of Quantum Mechanics," Springer Online Journal Archives, Vol. 65, No. 2,
1979, pp. 189-201.

[33] J. Jost, "Riemannian Geometry and Geometric Analysis," 4th Edition, Springer-Verlag, Berlin, 2005.

[34] Y. Makeenko, "Methods of Contemporary Gauge Theory," Cambridge University Press, Cambridge, 2002. http://dx.doi.org/10.1017/CBO9780511535147

[35] F. Bulnes, "Treatise of Advanced Mathematics: System and Signals Analysis," 1998.

[36] F. Bulnes and H. F. Bulnes, "Quantum Medicine Actions: Programming Path Integrals on Integral Mono-Pharmacists for Strengthening and Arranging of the Mind on Body," Journal of Frontiers of Public Health, Vol. 1, No. 3, 2012, pp. 57-65. http://dx.doi.org/10.5963/PHF0103001

[37] F. Bulnes, "Analysis and Design of Algorithms to the Master in Applied Informatics," Research Course, University of Informatics Sciences (UCI), Habana, 2007.

[38] F. Bulnes, "Cohomology of Cycles and Integral Topology," 2008. www.Appliedmath4.ipn.mx

[39] B. Simon and M. Reed, "Mathematical Methods for Physics," Vol. 1, Academic Press, New York, 1972.

[40] F. Bulnes, "Conferences of Mathematics: Seminar of Representation Theory of Reductive Lie Groups," Compilation of Institute of Mathematics, UNAM Publications, Mexico, 2000.

[41] F. Bulnes, "Handle of Scientific and Philosophical Methods of the Research: Guide of Prospective and Quality of the International Scientific and Technological Research," 2011.

[42] J. Dieudonne, "Treatise on Analysis," Vol. 6, Academic Press, New York, 1976.

[43] C. Truesdell and R. A. Topin, "The Classical Field Theories (in Encyclopaedia of Physics, Vol. III/1)," SpringerVerlag, Berlin, 1960. 